Zhao, J.; Özgen-Xian, I.; Liang, D.; Wang, T.; Hinkelmann, R.

\title{
A depth-averaged non-cohesive sediment transport model with improved discretization of flux and source terms
}

Journal article | Accepted manuscript (Postprint)

This version is available at https://doi.org/10.14279/depositonce-8417

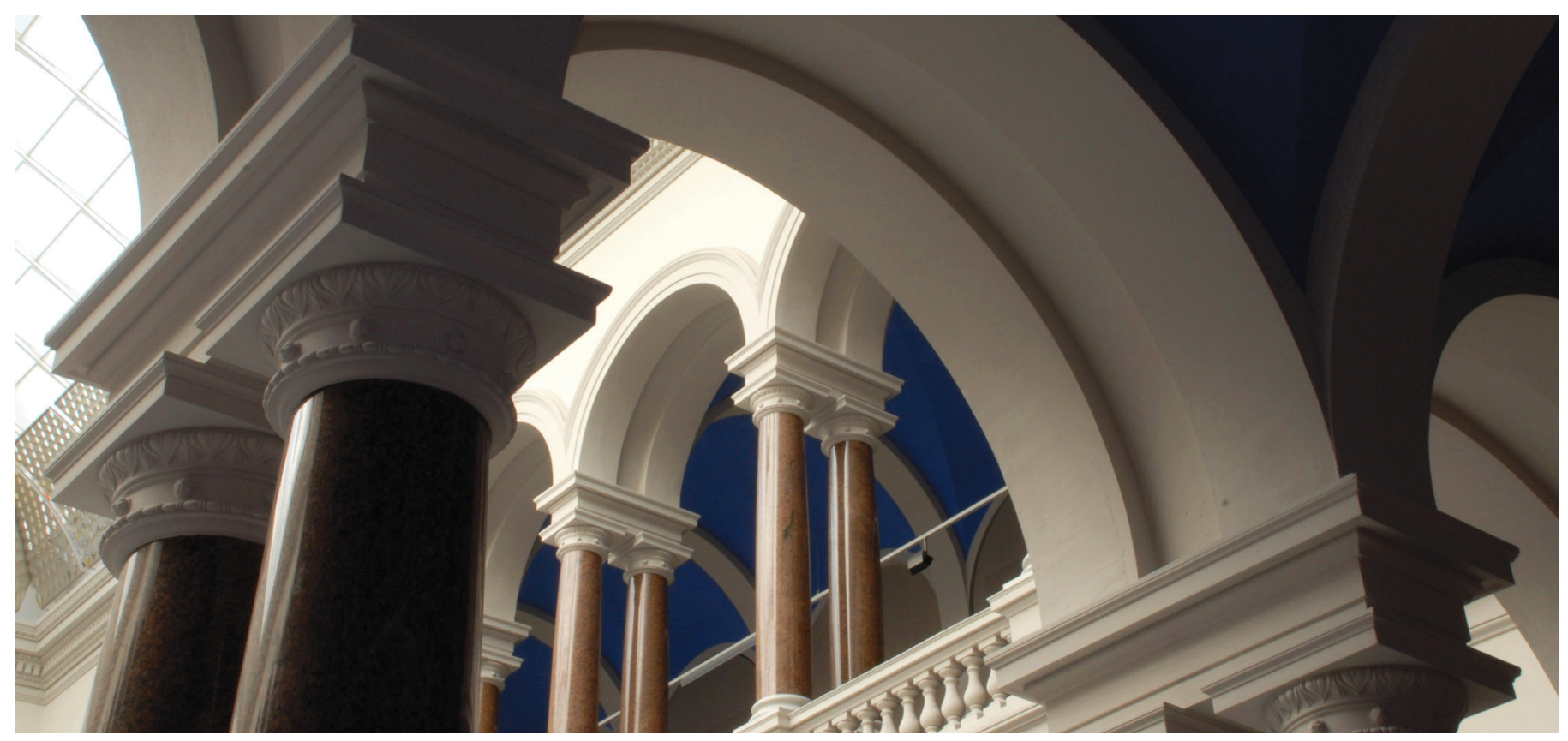

Zhao, J., Özgen-Xian, I., Liang, D., Wang, T., \& Hinkelmann, R. (2019). A depth-averaged non-cohesive sediment transport model with improved discretization of flux and source terms. Journal of Hydrology, 570, 647-665. https://doi.org/10.1016/j.jhydrol.2018.12.059 


\title{
A depth-averaged non-cohesive sediment transport model with improved discretization of flux and source terms
}

\author{
Jiaheng Zhao 1,*, Ilhan Özgen-Xian ${ }^{2}$, Dongfang Liang ${ }^{3}$, Tian \\ Wang $^{1,4}$, and Reinhard Hinkelmann ${ }^{1}$ \\ ${ }^{1}$ Chair of Water Resources Management and Modeling of Hydrosystems, \\ Technische Universität Berlin, Germany \\ ${ }^{2}$ Energy Geosciences Division, Lawrence Berkeley National Laboratory, US \\ ${ }^{3}$ Department of Engineering, University of Cambridge, Cambridge, UK \\ ${ }^{4}$ State Key Laboratory of Eco-hydraulics in Northwest Arid Region of China, \\ Xi'an University of Technology, Xi'an 710048, China \\ *Corresponding author: jiaheng.zhao@wahyd.tu-berlin.de \\ Preprint submitted to Journal of Hydrology, \\ final publication doi: 10.1016/j.jhydrol.2018.12.059
}

\begin{abstract}
This paper presents novel flux and source term treatments within a Godunov-type finite volume framework for predicting the depthaveraged shallow water flow and sediment transport with enhanced the accuracy and stability. The suspended load ratio is introduced to differentiate between the advection of the suspended load and
\end{abstract}


the advection of water. A modified Harten, Lax and van Leer Riemann solver with the contact wave restored (HLLC) is derived for the flux calculation based on the new wave pattern involving the suspended load ratio. The source term calculation is enhanced by means of a novel splitting-point implicit discretization. The slope effect is introduced by modifying the critical shear stress, with two treatments being discussed. The numerical scheme is tested in five examples that comprise both fixed and movable beds. The model predictions show good agreement with measurement, except for cases where local three-dimensional effects dominate.

sediment transport; total load model; HLLC Riemann solver; finite-volume method; source term treatment

\section{Highlights}

1. A second-order finite-volume method is presented for solving the total-load sediment transport

2. An improved HLLC Riemann solver is derived

3. An improved bed slope treatment is derived to account for density variation inside the cell

4. A novel implicit source term discretization is presented

5. The numerical model shows good agreement with measurement as long as the shallow flow assumptions are valid 


\section{Contents}

1 Introduction 4

2 Governing equations $\quad 8$

2.1 Hydrodynamic module . . . . . . . . . . . . . 9

2.2 Morphodynamic module . . . . . . . . . . . 10

3 Numerical scheme $\quad 13$

3.1 Novel HLLC approximate Riemann solver . . . . . . . . . . 15

3.2 Source term treatment . . . . . . . . . . . . . . . 22

3.3 MUSCL reconstruction . . . . . . . . . . . . 26

3.4 Boundary conditions . . . . . . . . . . 27

4 Computational examples $\quad 27$

4.1 Laboratory dam-break wave over a triangular bottom sill . 28

4.2 One-dimensional dam-break over movable bed . . . . . . . 31

4.3 Dyke erosion due to flow overtopping . . . . . . . . . . 39

4.4 Two-dimensional dam-break flow in a mobile channel with a sudden enlargement . . . . . . . . . . . . 4 49

4.5 Partial dam-break flow on movable bed in a straight channel 53

5 Conclusions

6 Acknowledgement 


\section{Introduction}

Flow processes often are associated with the transport of sediments, which impacts the topography of the earth. Sediment transport governs the erosion and deposition processes, the movement of sediment with fluid is among the most complex and least understood processes in nature [55]. Depending on its transport mode, sediment can be categorized as "suspended load" and "bed load". Here, suspended load describes the smaller particles that are suspended in the water, while the bed load is comprised of larger particles that are transported on the bed by means of rolling, sliding, or saltation. The mathematical and numerical modeling of these processes is challenging, because the erosion and deposition processes lead to a time-variable bottom elevation, which in return influences the flow. Current process-based sediment transport models use partial differential equations that are referred to as conservation laws to describe flow and transport processes [3, 25]. Usually, the water flow is solved by using either a kinematic or diffusive wave approximation, or by using the fully dynamic shallow water equation. The latter usually provide more accurate and detailed flow fields [7, 23, 24, 31, 35-37, 40, 43, 53, 57,60]. Based on the way the sediment transport is related to the flow, sediment transport models can be categorized into (1) decoupled and (2) coupled models. Decoupled flow and sediment transport models have been widely used in many real-life engineering problems. They are relatively easy to implement, and the results may be justified due to different time scales in flow and sediment transport and the using of empirical formulas for bed roughness and sediment transport capacity [55]. Most of the decoupled models are 
related to the equilibrium sediment transport assumption considering low sediment concentration and small bed change in each time step.

Fully coupled models that account for the coupling of water and sediment phases can be used at a wider range of flow conditions. These models are categorized as (1) Exner equation coupled models (bed load flux coupled model), e.g. [27, 32, 35, 36, 43], and (2) concentration flux coupled models, e.g. [7, 14-16,40,58,61]. The Exner equation coupled model solves the depth-averaged shallow water equations together with the Exner equation, which describes the sediment transport based on bed load movement through a power law for the flow velocity. The interaction between flow and sediment is accounted for by a variable parameter [23, 31, 35-37, 43]. Existing literature about the Exner equation treats the hydrodynamic and sediment mass conservation separately, without considering the influence of sediment movement on hydrodynamics [22, 31, 32,43]. This approach assumes that the movement of the sediment is much slower than the flow velocity. The concentration flux coupled model describes the sediment transport as a fully mixed suspended load, while the erosion and deposition processes are calculated with empirical equations. The sediment is modelled as a concentration in the water column, and its fluxes are calculated based on this concentration. Additional parameters are introduced to calculate mass exchange between the dissolved sediment and the bed, and additional source terms are introduced to account for the interaction between the sediment and flow $[7,40,57,60]$. The difference between the concentration flux coupled model and Exner equation coupled model is analyzed in Zhao et. al. [63]. The concentration flux coupled model is suggested for rapidly varying flows such as dam-break and tsunami. The Exner 
equation coupled model is more suitable for less varying flow such as river channel flow and overtopping flow.

Guan et. al. [15] propose a one-dimensional shallow water model coupled with sediment transport, which considers the velocity difference between the sediment and water flow. The model treats the sediment transport separately as bed load and suspended load. This model provides a way to simulate the sediment transport more physically, and it is suitable for more complex and different conditions. However, it is observed that even if the model in [15] uses different velocities for sediment transport and water flow, it neglects the influence of this difference on the Jacobian matrix, and the unmodified HLLC Riemann solver [50] was used to compute the numerical flux. Using the unmodified HLLC Riemann solver in this case is not optimal, because it neglects the additional wave emerging due to the difference in sediment and fluid velocities, and therefore calculates a non-optimal numerical flux.

In Audusse and Bristeau [2], a hydrostatic reconstruction of the bottom elevation is proposed that ensures non-negativity of water depth and preserves the C-property (i.e. if water level is constant, the momentum should equal to nil in the stationary case) [4] of the numerical scheme. This method uses the divergence form of the bed slope source, and shifts it to the cell edges [2]. In second-order schemes, the sediment concentration is interpolated linearly from cell center to the interface, which leads to a variation of density inside the cell. Hence, the density of the sediment flow mixture will be not distributed homogeneously, and the original treatment of the slope source will not provide a satisfying result anymore.

In order to avoid instability and spurious velocity due to stiff friction 
source terms for very shallow water depths, the friction source term can be discretized using the splitting point implicit treatment [6]. However, common sediment transport models in the literature usually discretize the source terms in an explicit way. This influences the stability of these schemes.

This work extends the idea of the multimode total load transport model of Guan et. al. [15] to present a two-dimensional, non-equilibrium, total load sediment transport model with several improvements in the numerical solution. In the proposed model, the bottom elevation is updated via the summation of erosion and deposition calculated by empirical equations based on the sediment concentration and flow field variables at the last time step. Sediment (include both suspended and bed load) is distributed into the water column represented by the sediment volume concentration. Sediment fluxes across the cell edges are transported as an additional transport term added to the shallow water equations. At the end of each time step, the concentration is updated by the sediment fluxes from the neighboring cells and the erosion and deposition inside the considered cell. In this process, the flow field is also influenced by sediment movement. The aforementioned shortcomings of existing sediment transport models are addressed as follows: (1) We derive a modified HLLC Riemann solver that accounts for the additional wave generated by the velocity difference between fluid and sediment; (2) We present an extension to the hydrostatic reconstruction [2] that accounts for variable density inside the computational cell. This ensures that the C-property of the numerical scheme is preserved and positive water depth reconstruction is guaranteed; (3) We utilize the splitting point implicit treatment [6] to discretize the additional source terms re- 
lated to sediment transport. This relaxes the time step restriction and improves the robustness of the scheme for small water depths. A robust shallow water total-load sediment transport model is finalized and solved based on the aforementioned novel numerical treatment, which provide a physical meaningful and numerical stable model to simulate the sediment transport with fluid.

Finally, we note that this work, similar to the work in [15], assumes that the sediment material is non-cohesive and turbulent effects are neglected. The implications of these assumptions are discussed in the conclusions.

\section{Governing equations}

The model consists of two modules that interact with each other via source terms; the hydrodynamic module and the morphodynamic module. The governing equations introduce a coefficient $\xi$ addressing the sediment to flow velocity, which is the ratio between the velocities of sediment advection and fluid movement. Although in [7,40,43] it is assumed that the flow velocity equals the sediment advection velocity, i.e. $\xi=1$, in this work these velocities are assumed to be different. With this additional velocity of sediment, the Jacobian matrix will change to reflect the different eigenstructure of the governing equations. Hence, a novel Riemann solver is derived to approximate the interfacial fluxes correctly. 


\subsection{Hydrodynamic module}

The hydrodynamic module considers the sediment-laden surface water flow that drives the bed evolution. The depth-averaged two-dimensional shallow water and sediment transport equations are used to describe the mass and momentum exchange of the sediment-water mixture flow [7, 40,61]. In order to account for the effect of the density change and bed evolution on the momentum of the flow, additional terms are added to the equations. The usual depth-averaged shallow flow assumptions are adopted here, i.e. the vertical acceleration of flow is negligible and the pressure is hydrostatic.

This yields the following equations:

$$
\begin{aligned}
\frac{\partial h}{\partial t}+\frac{\partial(h u)}{\partial x}+\frac{\partial(h v)}{\partial y} & =-\frac{\partial z_{b}}{\partial t} \\
\frac{\partial(h u)}{\partial t}+\frac{\partial\left(h u^{2}+\frac{1}{2} g h^{2}\right)}{\partial x}+\frac{\partial(h u v)}{\partial y} & =g h\left(S_{b x}+S_{f x}\right)-\frac{\rho_{s}-\rho_{w}}{2 \rho_{m}} g h^{2} \frac{\partial c}{\partial x} \\
& +\frac{\rho_{s}-\rho_{w}}{\rho_{m}} \frac{u \partial z_{b}}{\partial t} \xi(1-p-c) \\
\frac{\partial(h v)}{\partial t}+\frac{\partial(h u v)}{\partial x}+\frac{\partial\left(h v^{2}+\frac{1}{2} g h^{2}\right)}{\partial y} & =g h\left(S_{b y}+S_{f y}\right)-\frac{\rho_{s}-\rho_{w}}{2 \rho_{m}} g h^{2} \frac{\partial c}{\partial y} \\
& +\frac{\rho_{s}-\rho_{w}}{\rho_{m}} \frac{v \partial z_{b}}{\partial t} \xi(1-p-c),
\end{aligned}
$$

where $t, x$ and $y$ are time and two-dimensional Cartesian coordinates, $h$ is the water depth, and $u$ and $v$ are the velocity in $x$ - and $y$ - direction, respectively. $\left(S_{b x}, S_{b y}\right)$ and $\left(S_{f x}, S_{f y}\right)$ are the bed slope and friction source terms, $S_{b x}=-\partial z_{b} / \partial x, S_{b y}=-\partial z_{b} / \partial y, S_{f x}=C_{f} u \sqrt{u^{2}+v^{2}}$, $S_{f y}=C_{f} v \sqrt{u^{2}+v^{2}}, C_{f}$ is the bed roughness coefficient determined by the Manning coefficient $n$ and $h$ in the form of $g n^{2} / h^{1 / 3}, g$ represents the gravity acceleration, $\partial z_{b} / \partial t$ represents the rate of the bed elevation change, $\xi$ is the aforementioned sediment to flow velocity coefficient for 
total sediment transport that is calculated as

$$
\xi=\alpha / \beta+(1-\alpha)
$$

where $\alpha$ is the sediment transport mode parameter in the range of 0 to 1 which specifies the ratio of the bed load in total load, $\beta$ is the ratio of the fluid velocity relative to bed load velocity, and the velocity of the suspended load is assumed to be the same with the flow velocity. Values for $\alpha$ and $\beta$ can be obtained from [14], $p$ is the porosity of bed material. The last two terms on the right hand sides in Eq. (2) and (3) account for the spatial variations in sediment concentration and the momentum transfer between flow and erodible bed because of the sediment exchange and velocity difference between flow and bed material. $\rho_{m}$ is the depthaveraged density of sediment water mixture, $\rho_{w}$ and $\rho_{s}$ are the density of water and sediment, respectively, which can be calculated as

$$
\rho_{m}=\rho_{s} c+\rho_{w}(1-c)
$$

where $c$ is the depth-averaged volume concentration.

\subsection{Morphodynamic module}

The morphodynamic module considers sediment transport and bed evolution. These processes are governed by the suspended load and bed load equations. In [15], the suspended load model sets the advection velocity of the sediment equal to the flow velocity. The bed evolution is governed by

$$
\frac{\partial z_{b}}{\partial t}=\left[\alpha \frac{q_{b}-q_{b *}}{L_{a}}+(1-\alpha)(D-E)\right] /(1-p),
$$


and the sediment concentration is calculated by

$$
\frac{\partial h c}{\partial t}+\xi \frac{\partial h u c}{\partial x}+\xi \frac{\partial h v c}{\partial y}=-\frac{\partial Z_{b}}{\partial t}(1-p) .
$$

$D$ and $E$ are the deposition and entrainment fluxes representing the settling and entrainment of sediment respectively due to the suspended load transport. $q_{b}=\xi \sqrt{q_{x}^{2}+q_{y}^{2}} c$ is the bed load sediment transport rate $\left(\mathrm{m}^{2} / \mathrm{s}\right)$, where $q_{x}=u h$ and $q_{y}=v h$ are the unit width discharge $\left(\mathrm{m}^{2} / \mathrm{s}\right)$ in $x$ - and $y$-direction, and $q_{b *}$ is the bed load transport capacity $\left(\mathrm{m}^{2} / \mathrm{s}\right)$. Based on the non-equilibrium assumption, $L_{a}$ is the adaptation length of sediment $(\mathrm{m})$, which is the characteristic distance for sediment to recover from non-equilibrium transport towards equilibrium transport.

The widely used Meyer-Peter-Müller formula [34] is adopted to calculate the bed load transport capacity as

$$
q_{b *}=\varepsilon 8.0 \sqrt{\left(\frac{\rho_{s}}{\rho_{w}}-1\right) g d^{3}}\left(\theta-\theta_{c}\right)^{3 / 2},
$$

where $\varepsilon$ is a calibration parameter for erosion, $\theta$ and $\theta_{c}$ are, respectively, the real dimensionless bed shear stress and the critical dimensionless bed shear stress with $\theta=u_{*}^{2} /\left[\left(\rho_{s} / \rho_{w}-1\right) g d\right], d$ is the sediment diameter, $u_{*}=n \sqrt{g\left(u^{2}+v^{2}\right)} / h^{1 / 6}$ is the friction velocity, and $\theta_{c}$ can be related to following the empirical equation in [46]

$$
\theta_{c f}=\frac{0.3}{1+1.2 d_{*}}+0.055\left(1-e^{-0.02 d_{*}}\right)
$$

where $d_{*}=d_{50}\left[\left(\rho_{s} / \rho_{w}-1\right) g / \nu^{2}\right]^{1 / 3}$ is the dimensionless particle diameter, where $d_{50}$ is the median diameter. Considering the effect of longitudinal slopes, an empirical function is proposed in [41] as

$$
\frac{\theta_{c}}{\theta_{c f}}=\cos \varphi\left(1-\tan \varphi / \tan \varphi_{r}\right) .
$$


where $\theta_{c f}$ is the critical shear stress on the flat bottom calculated using Eq. (9), $\varphi_{r}$ is the repose angle, $\varphi$ is the bed slope angle, with positive values for down-slope beds. And a slope effect function from [9] is chosen for comparison as

$$
\frac{\theta_{c}}{\theta_{c f}}=\frac{\sin \left(\varphi_{r}-\varphi\right)}{\sin \varphi_{r}},
$$

The definition of the parameters is the same as in Eq. (11).

Deposition and entrainment fluxes of suspended load are calculated as $D=\omega_{s} C_{a}$ and $E=\omega_{s} C_{a e}$ [55]. $\omega_{s}$ settling velocity of naturally sediment particle $(\mathrm{m} / \mathrm{s})$ estimated as shown in [62]:

$$
\omega_{s}=\sqrt{\left(13.95 \frac{\nu}{d}\right)^{2}+1.09\left(\frac{\rho_{s}}{\rho_{w}}-1\right) g d}-13.95 \frac{\nu}{d}
$$

where $\nu$ is the water viscosity. $C_{a}=\phi c$, herein, $\phi=\min (2.0,(1-p) / c)$ is a parameter which depends on the distribution of the sediment over water column originally proposed in [7]. $C_{a e}$ is the near bed equilibrium concentration at a reference level $\sigma$ [15] above the bed, determined by the function proposed in [10] as

$$
C_{a e}=\frac{1}{11.6} \frac{q_{b *}}{\sigma U_{*}^{\prime}}
$$

where $U_{*}^{\prime}$ is the effective bed shear velocity related to grain roughness, determined by $U_{*}^{\prime}=U g^{0.5} / C_{h}^{\prime}$ with $C_{h}^{\prime}=18 \log (4 h / d)$, the reference level is chosen as $\sigma=2 d$.

In this work, sediment transport mode coefficient $\alpha$ is calculated by following an equation originally proposed in [14] as

$$
\begin{aligned}
& \alpha=1.0-\min \left(1,2.5 e^{-Z}\right), \\
& Z=\frac{\omega_{s}}{\kappa u_{*}},
\end{aligned}
$$


where $\kappa$ is the von Kármán constant, and is assumed equal to 0.41.

The first term of right hand side of Eq. (14) is the source term from bed load transport. For the bed load movement, it is assumed that the velocity difference is innegligible, which is supported by findings in $[14,52]$. In this work, the equation from [14] is used to estimate the appropriate velocity ratio for weak bed shear stress. For high bed shear stress with $\theta / \theta_{c r}>20$, the bed load velocity coefficient $\beta$ is set to be 1 , which yields

$$
\frac{1}{\beta}=\left\{\begin{array}{ll}
\frac{u_{*}}{u} \frac{1.1\left(\theta / \theta_{c}\right)^{0.17}\left[1-\exp \left(-5\left(\theta / \theta_{c}\right)\right)\right]}{\sqrt{\theta_{c}}} & \text { if } \theta / \theta_{c} \leq 20 \\
1 & \text { if } \theta / \theta_{c}>20
\end{array},\right.
$$

the adaption length $L_{a}$ has been studied in, e.g. [1, 14,55,56,59]. In this work, $L_{a}$ is calculated with

$$
L_{a}=\frac{h \sqrt{u^{2}+v^{2}}}{\gamma \omega_{s}}
$$

as described in [15], where $\gamma$ is the ratio of near bed concentration and volume concentration in flow. The value of $\gamma$ is calculated as

$$
\gamma=\min \left(\frac{h}{\beta h_{b}}, \frac{1-p}{c}\right),
$$

where the thickness of sheet-flow layer is calculated by the function $h_{b}=10 \theta d$ as proposed in [54].

\section{Numerical scheme}

Eq. (1), (2), (3), and (7) constitute a non-linear hyperbolic system. The governing equations can be rewritten in vector form as:

$$
\frac{\partial \mathbf{q}}{\partial t}+\frac{\partial \mathbf{f}}{\partial x}+\frac{\mathbf{g}}{\partial y}=\mathbf{s}
$$


with vectors define as:

$$
\begin{gathered}
\mathbf{q}=\left[\begin{array}{l}
h \\
h u \\
h v \\
c h
\end{array}\right], \quad \mathbf{f}=\left[\begin{array}{c}
h u \\
h u^{2}+g h^{2} / 2 \\
h u v \\
\xi u c h
\end{array}\right], \quad \mathbf{g}=\left[\begin{array}{c}
h v \\
h u v \\
h v^{2}+g h^{2} / 2 \\
\xi v c h
\end{array}\right], \\
\mathbf{s}=\left[\begin{array}{c}
\frac{\partial Z_{b}}{\partial t} \\
g h\left(S_{b x}+S_{f x}\right)-\frac{\rho_{s}-\rho_{w}}{2 \rho_{m}} g h^{2} \frac{\partial c}{\partial x}+\frac{\rho_{s}-\rho_{w}}{\rho_{m}} \frac{u \partial Z_{b}}{\partial t} \xi(1-p-c) \\
g h\left(S_{b y}+S_{f y}\right)-\frac{\rho_{s}-\rho_{w}}{2 \rho_{m}} g h^{2} \frac{\partial c}{\partial y}+\frac{\rho_{s}+\rho_{w}}{\rho_{m}} \frac{v \partial Z_{b}}{\partial t} \xi(1-p-c) \\
\alpha \frac{q_{b *}-q_{b}}{L_{a}}+(1-\alpha)(E-D)
\end{array}\right] .
\end{gathered}
$$

$\mathbf{q}$ is the vector of conserved variables, $\mathbf{f}$ and $\mathrm{g}$ are the flux vectors in $x-$ and $y$ - direction, respectively. $\mathrm{s}$ is the source term including the bed friction, bed slope and the additional terms associated with the sediment transport and bed deformation.

Eq. (19) can be written in integral form as:

$$
\int_{\Omega} \frac{\partial \mathbf{q}}{\partial t} d \Omega+\int_{\Omega}\left(\frac{\partial \mathbf{f}}{\partial x}+\frac{\partial \mathbf{g}}{\partial y}\right) d \Omega=\int_{\Omega} \mathbf{s} d \Omega
$$

where $\Omega$ is an arbitrary control volume (CV). Applying the Green-Gauß theorem and replacing the boundary integral with a sum over all edges, Eq. (20) becomes a finite-volume formulation written as

$$
\int_{\Omega} \frac{\partial \mathbf{q}}{\partial t} d \Omega+\sum_{k=1}^{m} \mathbf{F} \cdot \mathbf{n}_{k} l_{k}=\int_{\Omega} \mathbf{s} d \Omega
$$

where $m$ is the number of edges, $k$ is an index, and $\mathbf{n}=\left(n_{x}, n_{y}\right)^{T}$ is the unit vector in the outward direction normal to the interface of the cell, $l$ is the length of the edge, $\mathbf{F} \cdot \mathbf{n}$ is the flux vector normal to the interface 
and can be written as

$$
\mathbf{F} \cdot \mathbf{n}=\left(\mathbf{f n}_{\mathrm{x}}+\mathbf{g n}_{\mathrm{y}}\right)=\left[\begin{array}{c}
q_{x} n_{x}+q_{y} n_{y} \\
\left(u q_{x}+g h^{2} / 2\right) n_{x}+v q_{y} n_{y} \\
u q_{x} n_{x}+\left(v q_{y}+g h^{2} / 2\right) n_{y} \\
\xi q_{x} c n_{x}+\xi q_{y} c n_{y}
\end{array}\right] .
$$

The value of $q$ in cell $i$ is updated using the two-stage explicit RungeKutta scheme [21, 28, 29], where the value at the next time level in cell $i, \mathbf{q}_{i}^{n+1}$, is updated by

$$
\mathbf{q}_{i}^{n+1}=\frac{1}{2}\left\{\mathbf{q}_{i}^{n}+f\left[f\left(\mathbf{q}_{i}^{n}\right)\right]\right\}
$$

with

$$
f\left(\mathbf{q}_{i}^{n}\right)=\mathbf{q}_{i}^{n}+\frac{\Delta t^{n}}{\Omega}\left[\int_{\Omega} \mathbf{s}^{n+1} d \Omega-\sum_{k=1}^{m} \mathbf{F}\left(\mathbf{q}_{i}^{n}\right)_{k} \cdot \mathbf{n}_{k} l_{k}\right],
$$

where $\mathbf{s}^{n+1}$ is the source term composed with friction source and sediment movement discretized in a splitting point implicit way to be discussed in Sec. 3.2.2. $f()$ is a function to represent the updating process to a new time level in the considered cell. $\Delta t^{n}$ is the time step at the $n$th time level. For this work, the Courant-Friedrichs-Lewy condition is used here for maintaining the stability,

$$
\Delta t=\mathrm{CFL} \min \left(\frac{R_{1}}{\sqrt{u_{1}^{2}+v_{1}^{2}}+\sqrt{g h_{1}}}, \ldots, \frac{R_{n}}{\sqrt{u_{n}^{2}+v_{n}^{2}}+\sqrt{g h_{n}}}\right)
$$

where $R_{n}$ is the minimum distance from the cell center to the edge, CFL is the Courant-Friedrichs-Lewy number. For explicit time marching algorithms $\mathrm{CFL} \in(0,1]$. In this work, $\mathrm{CFL}=0.8$ is adopted.

\subsection{Novel HLLC approximate Riemann solver}

The introduction of the coefficient $\xi$ in Eq. (7) augments the Riemann solution with an additional contact wave. Fig. 1 shows a possible wave 


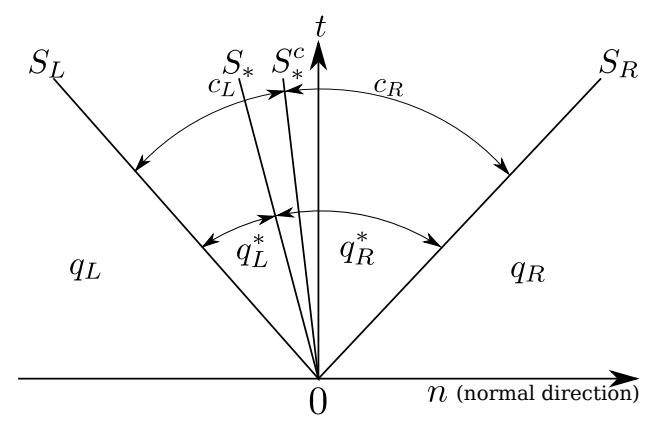

Figure 1: HLLC solution of the Riemann problem with $S_{L}, S_{*}, S_{*}^{c}, S_{R}$ describing the wave speed of the left wave, the contact waves for scalar and sediment and the right wave.

configuration for this Riemann problem. The wave propagating with the speed $S_{*}^{c}$ results from the introduction of $\xi$ and is distinct from the contact wave associated with the advection of the tangential velocity, which propagates with the speed $S_{*}$.

We now design a modified HLLC approximate Riemann solver that is suitable for the presented wave pattern. The presence of the source terms leads to a mixed system, but with the assumption of dominant advection it can be classified and numerically treated as a hyperbolic system [23]. Hence, from Eq. (21), a Jacobian matrix can be defined as $\mathbf{A}=\frac{\partial \mathbf{F} \cdot \mathbf{n}}{\partial \mathbf{q}}=\left[\begin{array}{cccc}0 & n_{x} & n_{y} & 0 \\ \left(-u^{2}+g h\right) n_{x}-u v n_{y} & 2 u n_{x}+v n_{y} & u n_{y} & 0 \\ -u v n_{x}+\left(-v^{2}+g h\right) n_{y} & v n_{x} & u n_{x}+2 v n_{y} & 0 \\ c \xi\left(-u n_{x}-v n_{y}\right) & \xi c n_{x} & \xi c n_{y} & \xi\left(u n_{x}+v n_{y}\right)\end{array}\right]$ 
The eigenvalues of the Jacobian matrix A can be obtained as:

$$
\left[\begin{array}{c}
\lambda_{1} \\
\lambda_{2} \\
\lambda_{3} \\
\lambda_{4}
\end{array}\right]=\left[\begin{array}{c}
u_{\perp}-a \\
u_{\perp} \\
u_{\perp}+a \\
\xi u_{\perp}
\end{array}\right]
$$

here, $u_{\perp}=u n_{x}+v n_{y}$ is the velocity normal to the interface, $a=\sqrt{g h}$ is the local dynamic wave velocity. There are 4 real and distinct eigenvalues, so the hyperbolicity of this system is preserved. We observe a 1-wave that is either a shock or a rarefaction, a 2-wave that is a contact wave, a 3-wave that is either a shock or a rarefaction and a 4-wave that is a contact wave. It can be thought to solve a one-dimensional Riemann problem across the cell interface in the normal direction of it. The tangential velocity is assumed to be transported with the mass flux. For sake of simplicity we consider the normal direction to be aligned with the $x$-axis, i.e. $\mathbf{n}=(1,0)$. The corresponding Jacobian matrix can be written as:

$$
\mathbf{A}_{\mathbf{s}}=\left[\begin{array}{cccc}
0 & 1 & 0 & 0 \\
a^{2}-u^{2} & 2 u & 0 & 0 \\
-u v & v & u & 0 \\
-c \xi u & \xi c & 0 & \xi u
\end{array}\right]
$$

where the velocity $u$ can be thought of as the velocity normal to the interface and $v$ is the tangential velocity. In order to analyze the RankineHugoniot condition across the shock waves and the generalized Riemann invariants across the rarefaction and contact waves, the right 
eigenvector of Jacobian $\mathbf{A}_{\mathbf{s}}$ can be calculated as:

$$
\mathbf{R}=\left[\begin{array}{cccc}
1 & 0 & 1 & 0 \\
u-a & 0 & u+a & 0 \\
v & 1 & v & 0 \\
\frac{-\xi c a}{u-a-\xi u} & 0 & \frac{\xi c a}{u+a-\xi u} & 1
\end{array}\right]
$$

The matrix $\mathbf{R}$ allows the following generalized Riemann invariants [49] to be defined for a solution made of simple waves:

$$
\begin{gathered}
\frac{\mathrm{d} h}{1}=\frac{\mathrm{d} q_{n}}{u-a}=\frac{\mathrm{d} q_{t}}{v}=\frac{\mathrm{d}(c h)}{\frac{-\xi c a}{u-a-\xi u}} \text { across } \frac{\mathrm{d} x}{\mathrm{~d} t}=u-a \\
\frac{\mathrm{d} h}{0}=\frac{\mathrm{d} q_{n}}{0}=\frac{\mathrm{d} q_{t}}{1}=\frac{\mathrm{d}(c h)}{0} \text { across } \frac{\mathrm{d} x}{\mathrm{~d} t}=u \\
\frac{\mathrm{d} h}{1}=\frac{\mathrm{d} q_{n}}{u+a}=\frac{\mathrm{d} q_{t}}{v}=\frac{\mathrm{d}(c h)}{\frac{\xi c a}{u+a-\xi u}} \text { across } \frac{\mathrm{d} x}{\mathrm{~d} t}=u+a \\
\frac{\mathrm{d} h}{0}=\frac{\mathrm{d} q_{n}}{0}=\frac{\mathrm{d} q_{t}}{0}=\frac{\mathrm{d}(c h)}{1} \text { across } \frac{\mathrm{d} x}{\mathrm{~d} t}=\xi u
\end{gathered}
$$

After integration, constant variables across simple waves lead to the following relationships:

$$
\begin{aligned}
& \begin{cases}u+2 a & =\text { const } \\
v & =\text { const, across } \frac{\mathrm{d} x}{\mathrm{~d} t}=u-a \\
\frac{c h}{[a+(\xi-1) u]^{2 \xi}} & =\text { const }\end{cases} \\
& \left\{\begin{aligned}
h & =\text { const } \\
q_{n} & =\text { const, across } \frac{\mathrm{d} x}{\mathrm{~d} t}=u \\
c h & =\text { const }
\end{aligned}\right. \\
& \begin{cases}u-2 a & =\text { const } \\
v \quad & \text { const, across } \frac{\mathrm{d} x}{\mathrm{~d} t}=u+a \\
\frac{c h}{[a+(1-\xi) u]^{2 \xi}} & =\text { const }\end{cases}
\end{aligned}
$$




$$
\begin{cases}h & =\text { const } \\ q_{n} & =\text { const, } \text { across } \frac{\mathrm{d} x}{\mathrm{~d} t}=\xi u \\ q_{t} & =\text { const }\end{cases}
$$

Consequently, in Eq. (35), $u=q_{n} / h$ also is constant across the wave, and $u=q_{n} / h, v=q_{t} / h$ are constant in Eq. (37), representing the contact discontinuity wave for $q_{t}$ and $c h$, respectively.

Based on a two rarefaction wave approximation [48], the immediate dynamic wave velocity $a_{*}$ can be obtained as

$$
a_{*}=\frac{1}{2}\left(a_{L}+a_{R}\right)-\frac{1}{4}\left(u_{R}-u_{L}\right)
$$

where $L$ and $R$ means the left and right side of the considered edge.

The corresponding velocity $u_{*}$ and water depth $h_{*}$ in the star region is given by

$$
\begin{gathered}
u_{*}=\frac{1}{2}\left(u_{L}+u_{R}\right)+a_{L}-a_{R}, \\
h_{*}=\frac{1}{g}\left[\frac{1}{2}\left(a_{L}+a_{R}\right)-\frac{1}{4}\left(u_{R}-u_{L}\right)\right]^{2} .
\end{gathered}
$$

Compared to the scalar transport equation in [48], the sediment concentration stays constant across the 1-, 2- and 3-wave, the water depth $h$ and the normal velocity $u$ change. The sediment concentration only changes across the 4 -wave, which is a contact wave. In the presented scheme, for the third terms in Eq. (34) and (36), it is assumed that the concentration $c$ stays constant. It is further assumed that the coefficient $\xi$ changes across the 1- and 3-wave, following a two shock wave approximation with two discontinuities. In the star region, the coefficient set to be a constant value $\xi_{*}$ (see Eq. (4)), i.e. it does not change across the 4-wave. 
With this knowledge about the physical problem, we calculate the wave speed $S_{*}$ by using the relationships in the star region defined in [49] as

$$
q_{* J}=h_{J}\left(\frac{S_{J}-u_{J}}{S_{J}-S_{*}}\right)\left[\begin{array}{c}
1 \\
S_{*} \\
u_{J}^{\|}
\end{array}\right]
$$

for $J=L, R$. For the wave speed $S_{*}^{c}$, the relationship can be written as

$$
q_{* J}=h_{J}\left(\frac{S_{J}-\xi_{J} u_{J}}{S_{J}-S_{*}^{c}}\right)\left[\begin{array}{c}
c_{J} \\
S_{*}^{c}
\end{array}\right] .
$$

Using the first components of the vectors in Eq. (41) and 42 each, and by noting that $h_{* L}=h_{* R}$, we obtain the two wave speeds as

$$
\begin{aligned}
S_{*} & =\frac{S_{L} h_{R}\left(u_{R}-S_{R}\right)-S_{R} h_{L}\left(u_{L}-S_{L}\right)}{h_{R}\left(u_{R}-S_{R}\right)-h_{L}\left(u_{L}-S_{L}\right)} \\
S_{*}^{c} & =\frac{S_{L} h_{R}\left(u_{R} \xi_{R}-S_{R}\right)-S_{R} h_{L}\left(u_{L} \xi_{L}-S_{L}\right)}{h_{R}\left(u_{R} \xi_{R}-S_{R}\right)-h_{L}\left(u_{L} \xi_{L}-S_{L}\right)} .
\end{aligned}
$$

The tangential velocity $u^{\|}$changes across the 2-wave propagating with the speed $S_{*}$ and the sediment concentration changes across the 4-wave propagating with the speed $S_{*}^{c}$.

The HLLC solution for the hydrodynamic module is

$$
F_{i+1 / 2}^{\text {hllc }}= \begin{cases}F_{L} & \text { if } 0 \leq S_{L} \\ F_{*, L} & \text { if } S_{L}<0 \leq S_{*} \\ F_{*, R} & \text { if } S_{*}<0 \leq S_{R} \\ F_{R} & \text { if } S_{R}<0\end{cases}
$$

where $S_{L}$ and $S_{R}$ are the 1- and 3-wave speeds, respectively, cf. Fig.1. They can estimated following [12] as:

$$
S_{L}= \begin{cases}u_{R}-2 \sqrt{g h_{R}} & \text { if } h_{L}=0 \\ \min \left(u_{L}-\sqrt{g h_{L}}, u_{*}-\sqrt{g h_{*}}\right) & \text { if } h_{L}>0\end{cases}
$$




$$
S_{R}=\left\{\begin{array}{ll}
u_{L}+2 \sqrt{g h_{L}} & \text { if } h_{R}=0 \\
\max \left(u_{R}+\sqrt{g h_{R}}, u_{*}-\sqrt{g h_{*}}\right) & \text { if } h_{L}>0
\end{array} .\right.
$$

The fluxes $\mathbf{F}_{L}$ and $\mathbf{F}_{R}$ are calculated from the left and right Riemann states, $\mathbf{q}_{L}$ and $\mathbf{q}_{R}$ respectively. As described in [45], the fluxes at the left and right side of the 2-wave, $\mathbf{F}_{*, L}$ and $\mathbf{F}_{*, R}$ are given by

$$
\begin{gathered}
F_{*, L}=\left[\begin{array}{c}
F_{*, 1} \\
F_{*, 2} n_{x}-u^{\|, L} F_{*, 1} n_{y} \\
F_{*, 2} n_{y}+u^{\|, L} F_{*, 1} n_{x}
\end{array}\right], \\
F_{*, R}=\left[\begin{array}{c}
F_{*, 1} \\
F_{*, 2} n_{x}-u^{\|, R} F_{*, 1} n_{y} \\
F_{*, 2} n_{y}+u^{\|, R} F_{*, 1} n_{x}
\end{array}\right] .
\end{gathered}
$$

The HLLC solution for the morphodynamic module is

$$
F_{4}=F_{i+1 / 2}^{s . h l l c}= \begin{cases}F_{L, 1} c_{L} & \text { if } 0 \leq S_{L} \\ F_{*, s} c_{L} & \text { if } S_{L}<0 \leq S_{*}^{c} \\ F_{*, s} c_{R} & \text { if } S_{*}^{c}<0 \leq S_{R} \\ F_{R, 1} c_{R} & \text { if } S_{R}<0\end{cases}
$$

where the tangential velocity $u^{\|}$is obtained with $u^{\|}=-u n_{y}+v n_{x}$. The flux in the star region of the hydrodynamic module is calculated by using the HLL flux equation [48] as

$$
F_{*}=\frac{S_{R} F\left(q^{\perp L}\right)-S_{L} F\left(q^{\perp R}\right)+S_{L} S_{R}\left(q^{\perp R}-q^{\perp L}\right)}{S_{R}-S_{L}}
$$

where the normal variables $q^{\perp}$ and the fluxes $F$ are calculated as

$$
q^{\perp}=\left[\begin{array}{c}
h \\
q_{x} n_{x}+q_{y} n_{y}
\end{array}\right], F\left(q^{\perp}\right)=\left[\begin{array}{c}
h u^{\perp} \\
u^{\perp}\left(q_{x} n_{x}+q_{y} n_{y}\right)+g h^{2} / 2
\end{array}\right],
$$


The HLL flux of the morphodynamic module, $F_{*, s}$, is calculated by using the following relationships:

$$
\begin{aligned}
& \xi_{L} u_{L}^{\perp} c_{L} h_{L}-F_{*, s} c_{L}=\left(\xi_{L} c_{L} h_{L}-\xi_{*} c_{L} h_{*}\right) S_{L} \\
& \xi_{R} u \frac{\perp}{R} c_{R} h_{R}-F_{*, s} c_{R}=\left(\xi_{R} c_{R} h_{R}-\xi_{*} c_{R} h_{*}\right) S_{R}
\end{aligned}
$$

The solution of this system of two equations with two unknowns is unique, and $F_{*, s}$ can be calculated as

$$
F_{*, s}=\frac{S_{R}\left(\xi_{L} u_{L}^{\perp} h_{L}\right)-S_{L}\left(\xi_{R} u \frac{\perp}{R} h_{R}\right)+S_{L} S_{R}\left(\xi_{R} h_{R}-\xi_{L} h_{L}\right)}{S_{R}-S_{L}} .
$$

This completes the presentation of the novel HLLC approximate Riemann solver.

\subsection{Source term treatment}

We propose an improved slope source term calculation based on the method in [2]. In order to prevent an overestimation of the source term, a splitting point implicit method is proposed to calculate the friction and sediment source terms.

\subsubsection{Improved slope source term treatment}

The slope treatment in [2] is modified to account for the density change due to suspended load. Variables at the cell edges are adjusted by using the non-negative water depth reconstruction from [20].

Slope terms in the cell are projected onto the edges using

$$
\int_{\Omega} S_{b} d_{\Omega}=\oint_{\Gamma} \mathbf{F}_{S M}(q) d \Gamma=\sum_{k=1}^{m}\left[\mathbf{F}_{S M}(q) l_{M}\right]
$$

where $\mathbf{F}_{S M}$ represents the flux vector of the slope source terms, located at the middle of the edge and along the normal direction of this edge, $M$ 


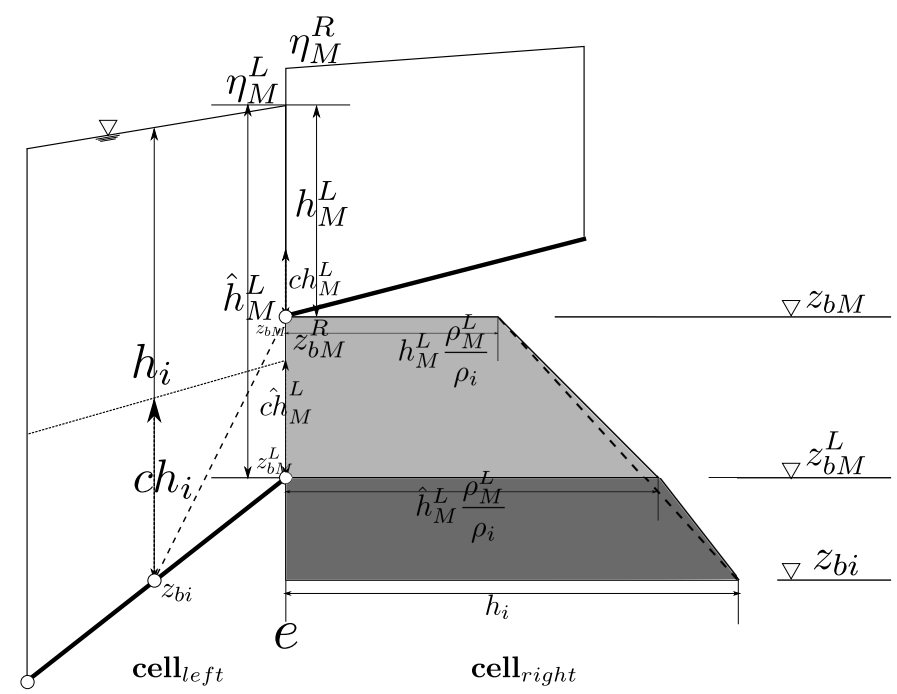

Figure 2: Improved slope source term treatment at the edge of $e$ of the left cell.

is the index of the edges, $l_{M}$ is the length of the edge, and $m$ is the total number of the edges in the considered cell.

As shown in Fig. 2, the slope source flux can be separated into an interface part that results from the hydrostatic reconstruction and a inner part due the results from the bed elevation change from the cell center to the edge center.

The calculation of the variables at the edge is based on the averaged variables inside the considered cell. Hence, the reconstruction at the edge can be enhanced by taking the density variation inside the cell into account. This can be achieved by multiplying the water depth with the ratio of the density at the edge, $\rho_{M}$, to the density at the cell center, $\rho_{i}$. The fluxes at the interface $F_{S M}^{I}$ and the center $F_{S M}^{C}$ can be written 
as

$$
\begin{aligned}
& \mathbf{F}_{S M}^{I}=\frac{g \rho_{M}^{L}}{2 \rho_{i}}\left[\left(h_{M}^{L}\right)^{2}-\left(\hat{h}_{M}^{L}\right)^{2}\right], \\
& \mathbf{F}_{S M}^{C}=-\frac{g}{2}\left(\frac{\rho_{M}^{L}}{\rho_{i}} \hat{h}_{M}^{L}+h_{i}\right)\left(z_{b M}^{L}-z_{b i}\right),
\end{aligned}
$$

and the normal flux of bed slope can be calculated as

$$
\mathbf{F}_{S M}(\mathbf{q})=\mathbf{F}_{S M} \mathbf{n}_{M}=\left(\mathbf{F}_{S M}^{I}+\mathbf{F}_{S M}^{C}\right) \mathbf{n}_{M},
$$

where $\mathbf{n}_{M}=\left(n_{x}, n_{y}\right)^{T}$ is the unit normal vector of the edge, $\hat{h}_{M}^{L}$ is the water depth after interpolation from the cell center, as shown in Fig.2, $z_{b i}, h_{i}$, and $c h_{i}$ are the bottom elevation, water depth and sediment volume depth at cell center, respectively, and similarly $z_{b M}^{L}, \hat{h}_{M}^{L}$, and $c \hat{h}_{M}^{L}$ are the bottom elevation, water depth and sediment volume depth after the interpolation but before the hydrostatic reconstruction, respectively, and finally, $h_{M}^{L}$ is the water depth after the interpolation and after the hydrostatic reconstruction.

We can introduce a virtual bed and ignore the influence of the water body under the virtual bed [21], which gives the slope flux that accounts for the density variation as

$$
\mathbf{F}_{S M}=\frac{g}{2}\left[-\left(\frac{\rho_{M}^{L}}{\rho_{i}} h_{M}^{L}+h_{i}\right)\left(z_{b M}-z_{b i}\right)\right],
$$

and the final slope flux is given by

$$
\mathbf{F}_{S M}=\left[\begin{array}{c}
0 \\
-\mathbf{n}_{x} \frac{g}{2}\left(\frac{\rho_{M}^{L}}{\rho_{i}} h_{M}^{L}+h_{i}\right)\left(z_{b M}-z_{b i}\right) \\
-\mathbf{n}_{y} \frac{g}{2}\left(\frac{\rho_{M}^{L}}{\rho_{i}} h_{M}^{L}+h_{i}\right)\left(z_{b M}-z_{b i}\right) \\
0
\end{array}\right] .
$$

At steady state with a homogeneous concentration, the density is constant and the ratio $\rho_{M}^{L} / \rho_{i}$ equals to 1 . Then, the slope flux is equivalent 
to the one presented in [21], which is proven to preserve the C-property. Hence, the presented numerical scheme is also well-balanced and Cproperty preserving.

\subsubsection{Splitting point implicit source term treatment}

We now focus on the discretization of the remaining source terms. The most straight-forward technique would be to treat them explicitly in time. However, this approach yields numerical instabilities unless the time step size $\Delta t$ satisfies [17]:

$$
-1 \leq 1+\frac{S\left(U_{i}^{n+1, x}\right)}{U_{i}^{n+1, x}} \Delta t \leq 1,
$$

where $U_{i}^{n+1, x}$ is the solution after adding the fluxes terms, and the time step has to be calculated using

$$
\begin{aligned}
\Delta t_{S} & =\operatorname{Min}_{i=1, \ldots, N}\left[-2 \frac{U_{i}^{n+1, x}}{S\left(U_{i}^{n+1, x}\right)}\right] \\
\Delta t & =\operatorname{Min}\left(\Delta t_{c}, \Delta t_{S}\right),
\end{aligned}
$$

where $\Delta t, \Delta t_{S}$ and $\Delta t_{c}$ are time steps for the system, source term part and conservation part, respectively. Depending on the source term, this might result in a severe degradation of the time step size.

To overcome this limitation, in literature, e.g. [20,21], the splitting point-implicit method is adopted. This avoids the instability of the numerical scheme for very shallow water depths.

In splitting point implicit methods, conserved variables inside the cell are updated as

$$
\mathbf{q}^{n+1}=\mathbf{q}^{n}+\frac{1}{\mathbf{P I}}\left(-\frac{\Delta t}{A} \sum_{k} \mathbf{f}_{k}^{n} \cdot \mathbf{n}_{k} l_{k}+\Delta t \mathbf{S}^{n}\right) .
$$


Here, $n$ and $n+1$ represent the time levels and $\mathbf{P I}$ is a matrix equal to

$$
\mathbf{P I}=\mathbf{I}-\Delta t\left(\frac{\partial \mathbf{S}}{\partial \mathbf{q}}\right)^{n}
$$

We now derive all momentum source terms with respect to the unit discharge, except the slope source term that has been transformed into fluxes over the cell edges. Eq. (66) then yields

$$
\mathbf{P I}=\left[1-\Delta t\left(\partial S_{x} / \partial q_{x}\right)^{n}, 1-\Delta t\left(\partial S_{y} / \partial q_{y}\right)^{n}\right]^{T}
$$

This gives

$$
\begin{aligned}
& \frac{\partial S_{x}}{\partial q_{x}}=-\frac{C_{f}}{h^{2}}\left(\hat{q}+\frac{q_{x}^{2}}{\hat{q}}\right)+\frac{\rho_{s}-\rho_{w}}{\rho_{m}} \frac{\partial z}{\partial t} \frac{\xi(1-p-c)}{h} \\
& \frac{\partial S_{y}}{\partial q_{y}}=-\frac{C_{f}}{h^{2}}\left(\hat{q}+\frac{q_{y}^{2}}{\hat{q}}\right)+\frac{\rho_{s}-\rho_{w}}{\rho_{m}} \frac{\partial z}{\partial t} \frac{\xi(1-p-c)}{h}
\end{aligned}
$$

where $\hat{q}=\sqrt{q_{x}^{2}+q_{y}^{2}}$ is the magnitude of the unit discharge vector.

\subsection{MUSCL reconstruction}

We use a TVD-MUSCL reconstruction of cell-averaged variables [51] to obtain second order accuracy. There are many TVD-MUSCL schemes in literature, cf. e.g. [5, 18, 21, 26, 30, 39, 49,64]. In this work, we apply the multislope total variation diminishing (TVD) scheme from [64].

If not treated properly, the MUSCL reconstruction will overestimate the sediment volume $c h$ at the cell interfaces, leading to concentrations larger than 1. We use the sediment diameter to limit the MUSCL reconstruction of $c h$ at cell interfaces as

$$
c_{i}= \begin{cases}(c h)_{i} / h_{i} & \text { if } h_{i}>d \\ (c h)_{e} / h_{e} & \text { if } h_{i} \leq d\end{cases}
$$


where, $c_{i},(c h)_{i}$, and $h_{i}$ represent the interpolated concentration, sediment volume and water depth, respectively, along the interface, and $c_{e},(c h)_{e}$, and $h_{e}$ are the corresponding values at the cell center. The threshold value for determining whether a cell is wet or dry is set to be $10^{-6} \mathrm{~m}$.

\subsection{Boundary conditions}

The hydrodynamic module uses the ghost cell-based boundary conditions presented in [21]. The sediment concentration is set

$$
c_{b}=c_{i}
$$

for all boundary conditions, with $c_{b}$ being the concentration of the ghost cells, and $c_{i}$ being the interpolated value of the shared interfaces.

\section{Computational examples}

A series of model tests were undertaken to verify the numerical model outlined above, the predictions of the proposed model will be compared the alternative numerical solutions and laboratory experimental data published in the literature. Five test cases of dam-break and dyke overtopping flows were undertaken, (i) a dam-break flow wave over a triangular bottom, (ii) one-dimensional dam-break over movable bed, (iii) dyke erosion due to flow overtopping, (iv) dam-break flow in a mobile channel with a sudden enlargement, and (v) a partial dam-break flow on movable bed in a straight channel.

Sensitivity analysis is investigated against one-dimensional dambreak over movable bed, four parameters which include Manning num- 
ber $n$, sediment diameter $d$, and sediment porosity $p$ are chosen to consider the sensitivity to the sediment movement. The root-mean-square error (RMSE) of the bottom is chosen to evaluate the difference of the simulation results,

$$
R M S E=\sqrt{\frac{\sum_{i=1}^{N}\left[\left(z_{b i}-z_{b i 0}\right)^{2} \Omega_{i}\right]}{\sum_{i=1}^{N} \Omega_{i}}}
$$

which, $N$ is the number of the cells, $z_{b i 0}$ is the benchmark bottom elevation for comparison.

In this work, the density of water is set to be $\rho_{w}=1000 \mathrm{~kg} / \mathrm{m}^{3}$, water viscosity is $\nu=1.2 \mathrm{e}-6$, and gravity $g=9.81 \mathrm{~m} / \mathrm{s}^{2}$, the sediment diameter $d$, density $\rho_{s}$, porosity $p$, repose angle $\varphi_{r}$ and the Manning number of the computational domain $n$ will be specified in each test case, the parameter $\varepsilon$ in Eq. (8) will be specified after calibration.

\subsection{Laboratory dam-break wave over a triangular bottom sill}

Aim of this test case is to verify the hydrodynamic module of the proposed scheme. A laboratory experiment considering a dam-break wave over a triangular bottom sill is reproduced. Measurement data, experimental setup and numerical parameters are provided in [44]. A sketch of the setup is shown in Fig. 3. There is a dam located at the $2.39 \mathrm{~m}$ of a $5.6 \mathrm{~m}$ long and $0.5 \mathrm{~m}$ wide horizontal channel, and a reservoir is formed at the upstream of the gate with a $0.111 \mathrm{~m}$ deep still water. A symmetrical bump is set at $x=4.45$ with a height of $0.065 \mathrm{~m}$ and bed slopes of \pm 0.14 . Between the bump and wall in downstream, a pool is set with an initial water level at $0.02 \mathrm{~m}$ above the flat bottom. Three gauges are installed to measure the water level around the bump, which are located 


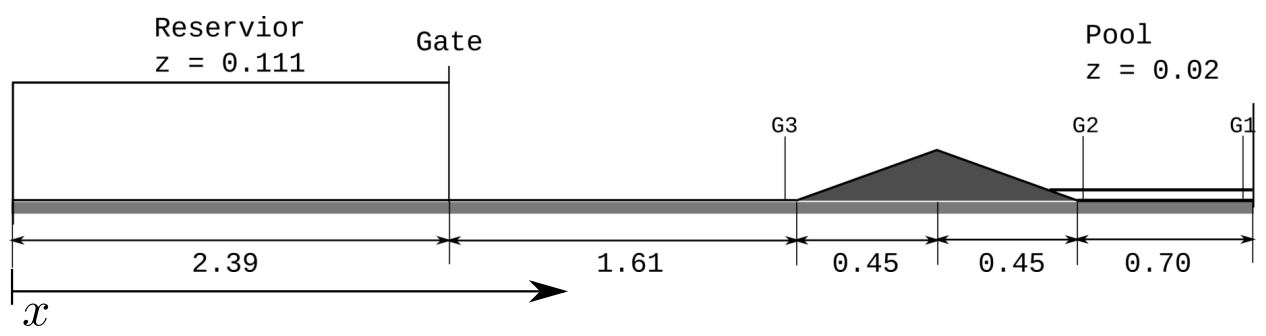

Figure 3: Dam-break over a triangular bottom sill: experimental setup and initial conditions (all dimensions are in $\mathrm{m}$ ) [44].

along the centreline of the channel with $x_{1}=5.575 \mathrm{~m}, x_{2}=4.925 \mathrm{~m}$ and $x_{3}=3.935 \mathrm{~m}$ for representing the location of $\mathrm{G} 1, \mathrm{G} 2$ and $\mathrm{G} 3$ respectively.

As this is a one-dimensional test case, for the sake of efficiency, the numerical solution is based on a $5.6 \mathrm{~m} \times 0.2 \mathrm{~m}$ computational domain. All boundary conditions are closed boundaries. The domain is discretized with 1400 cells. The simulation stops after 45 s. A Manning coefficient $n=0.011 \mathrm{sm}^{-1 / 3}$ is given as suggested in [44].

In this test case, the bed is fixed and therefore only the hydrodynamic module takes part in the calculation. All source terms and fluxes that are related to the morphodynamic module are automatically equal to zero. The computed water levels are compared with measurement data at three gauges are plotted in Fig. 4. Very good agreement between model results and measurement data is achieved.

As the sediment movement is mainly caused through exceeding the shear stress, which means that even on the fixed bed, the coefficients still can be calculated, and as there is no interaction between the flow and the sediment movement, it is straightforward to check the laws of the relationship between the coefficients. In order to show the sensitivity of the coefficient in this test case, a group of imaginary initial 

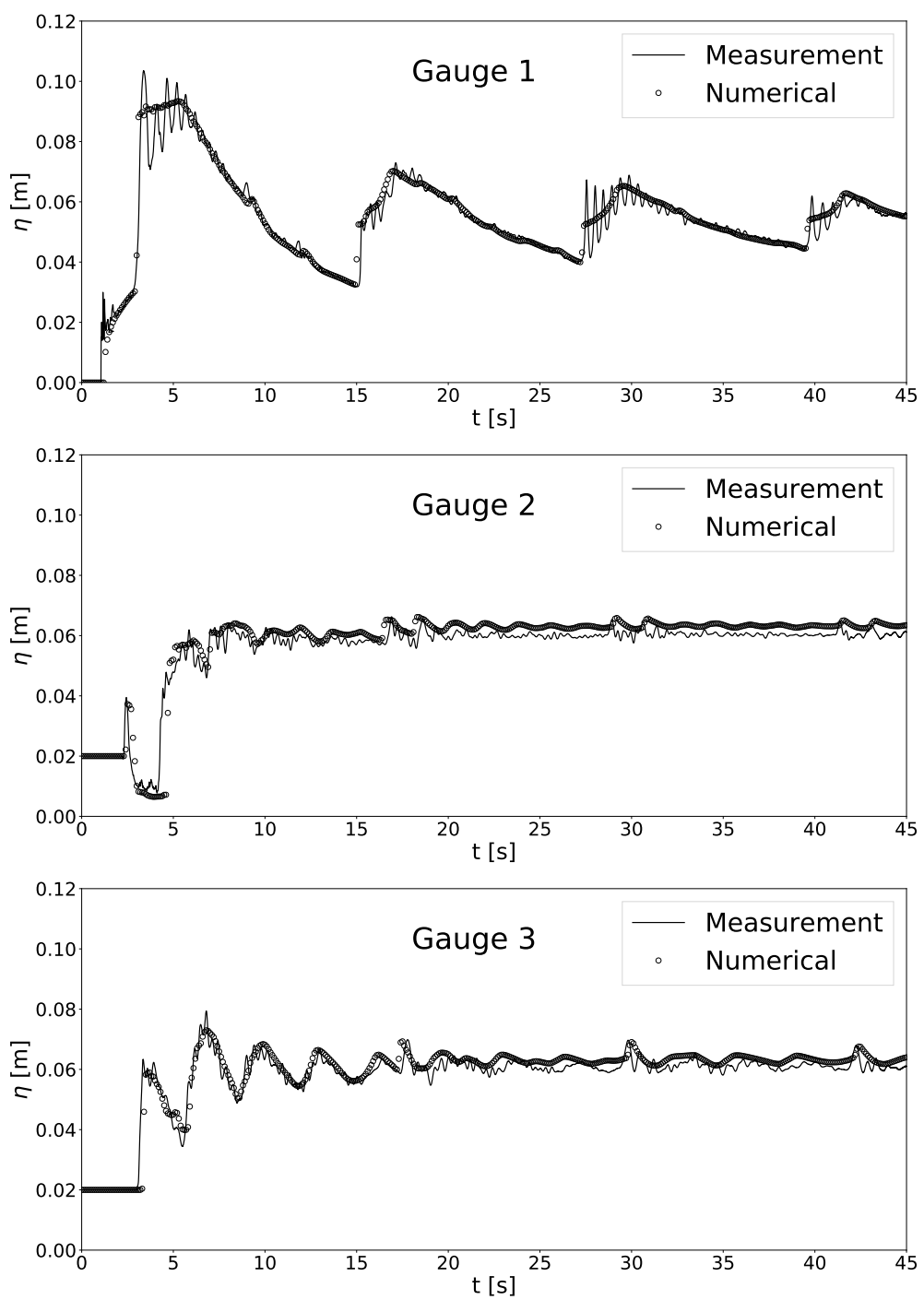

Figure 4: Dam-break over a triangular bottom sill: time histories of water levels at: (a) gauge 1, (b) gauge 2, (c) gauge 3 . 
conditions are studied for the sediment. Here, the sediment diameter is $d=0.008 \mathrm{~m}$, and the density is set to be $\rho_{s}=2650 \mathrm{~kg} / \mathrm{m}^{3}$, the porosity of sediment bed $p=0.4$, calibration parameter $\varepsilon=1.0$, and the repose angle is $\varphi_{r}=30^{\circ}$. The water levels around the triangular bump and coefficients for sediment transport at $1.8 \mathrm{~s}, 3.0 \mathrm{~s}$ and $8.4 \mathrm{~s}$ are plotted in Fig. 5. The water levels are well captured by the numerical simulation. The sediment velocity coefficient $\xi$ behaves similar to the suspended load coefficient $1-\alpha$. This is because $\xi$ is calculated based on the ratio of the suspended load coefficient to the bed load velocity coefficient $1 / \beta$, cf. Eq. (4). We note that $1 / \beta<1$, which means the more suspended load in the sediment transport, the larger the sediment velocity will be. Taking the partial derivative of Eq. (4) with respect to the ratio of suspended load $1-\alpha$, we obtain $\partial \xi / \partial(1-\alpha)=1-1 / \beta$, as shown in Eq. (16), $1 / \beta \leq 1.0$ which means that the sediment velocity is increasing with the ratio of suspended load.

\subsection{One-dimensional dam-break over movable bed}

\subsubsection{One-dimensional dam-break over movable bed test against experiment}

The purpose of this test case is to analyze the model parameters related to the morphodynamic module and assess the model performance for sediment transport for rapidly varying flow. A laboratory experiment that considers a dam-break wave over movable bed is reproduced numerically. The experimental data, initial conditions and model parameters can be found in [11]. The domain is $2.5 \mathrm{~m}$ long and $0.1 \mathrm{~m}$ wide. A dam is set at $1.25 \mathrm{~m}$. The upstream water depth is initially $h_{0}=0.1 \mathrm{~m}$, 

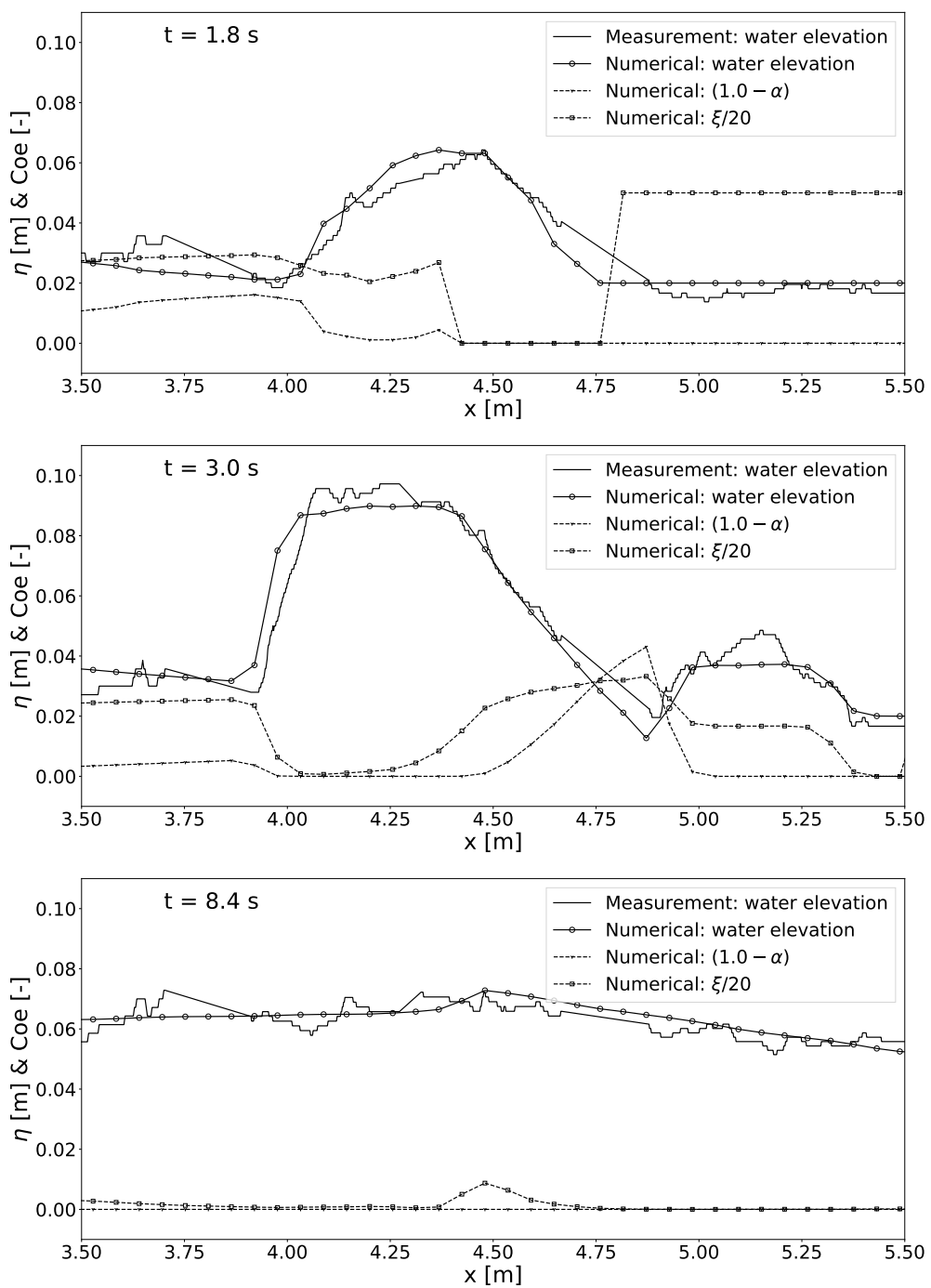

Figure 5: Dam-break over a triangular bottom sill: water level and coefficients around triangular bottom sill at: (a) $t=1.8 \mathrm{~s}$, (b) $t=3.0 \mathrm{~s}$, (c) $t=8.4 \mathrm{~s}$. 


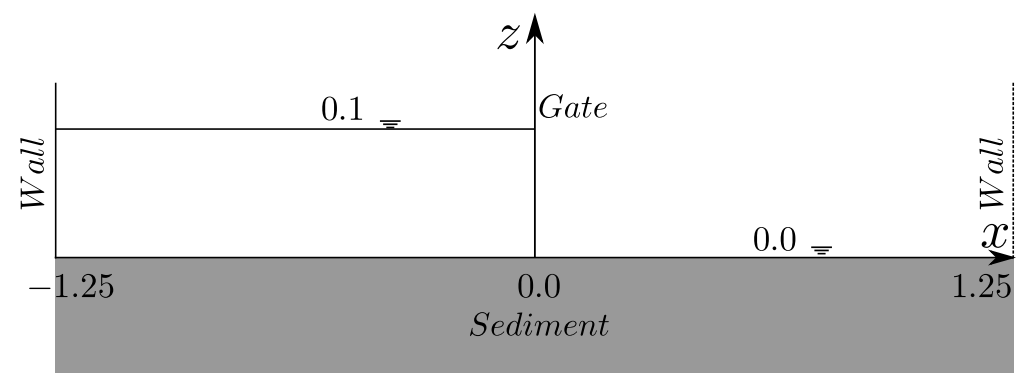

Figure 6: One-dimensional dam-break over movable bed: sketch of the experiment set up, initial and boundary conditions (dimension in meters).

and with dry bed downstream, four boundaries are set to be solid boundaries, there will be a hydraulic jump happen near to the location of the dam during the flow process. A sediment layer with a constant thickness of approximately $5-6 \mathrm{~cm}$ is placed within the boundaries domain, the sediment diameter is reported to $d=0.0035 \mathrm{~m}$, and the density is $\rho_{s}=1540 \mathrm{~kg} / \mathrm{m}^{3}$, bed porosity is $p=0.3$, the Manning coefficient $n=0.025 \mathrm{sm}^{-1 / 3}$, the repose angle $\varphi_{r}=30^{\circ}$, and the erosion calibration parameter $\varepsilon=2.4$. The domain is discretized with 1710 triangular cells, whole experiment runs for $2 \mathrm{~s}$.

Model results are compared with measurement data and a pseudoanalytical solution from [11]. Fig. 7 (a-c) shows the comparison of water levels and bed elevations. Overall good agreement is observed, the position of the largest erosion and its elevation are well predicted and the hydraulic jump is captured accurately. Compared to the pseudoanalytical results, the proposed model performs better with regard to water level prediction at the upstream of the dam-break. However, both of the water elevations for the hydraulic jump are not well captured 
by the proposed model and the pseudo-analytical model, this may due to the gate opening generated a localized disturbance on the nearby region, which the flow does not completely smooth out as it becomes shallower which lead to the non-hydrostatic effect arise in this region, breaks down the shallow water assumption. Here, the bed elevation is also predicted more accurately by the proposed model. The shock propagating in downstream direction is not captured well by the pseudoanalytical solution because it neglects the influence of the additional source terms due to sediment transport.

Due to the total load sediment transport concept of the proposed scheme the sediment is transported as suspended load and as bedload. The related coefficients are plotted in Fig. 8. We observe that large velocities yield large values of suspended transport ratio $(1-\alpha)$ (see Eq. (14)). Bed load transport dominants upstream while in the region near to the shock wave suspended load transport dominates.

Fig. 8 also shows that the velocity of the water sediment mixture column $u$ exhibits similar behavior as the suspended load ratio $(1-\alpha)$ (see Eq. (14)), Shield's parameter $\theta$ and the sediment concentration. Based on the Eq. (17) and Eq. (18), it can be observed that with the increasing of adaption length $L_{a}$, there is a monotonically increasing tendency for the flow velocity, Shield's parameter $\theta$, ratio of suspended load $1-\alpha$, and the sediment flux $\hat{q} c$. This relationship can be seen in Fig. 8, where the adaption length is the parameter used for sediment exchange from the non-equilibrium to equilibrium state. For high velocity and high concentration conditions, the corresponding adaption length will be longer. As the velocity of suspended load is assumed equal to the fluid, which means that sediment velocity coefficient $\xi$ (see Eq. (4)) is mainly depend 

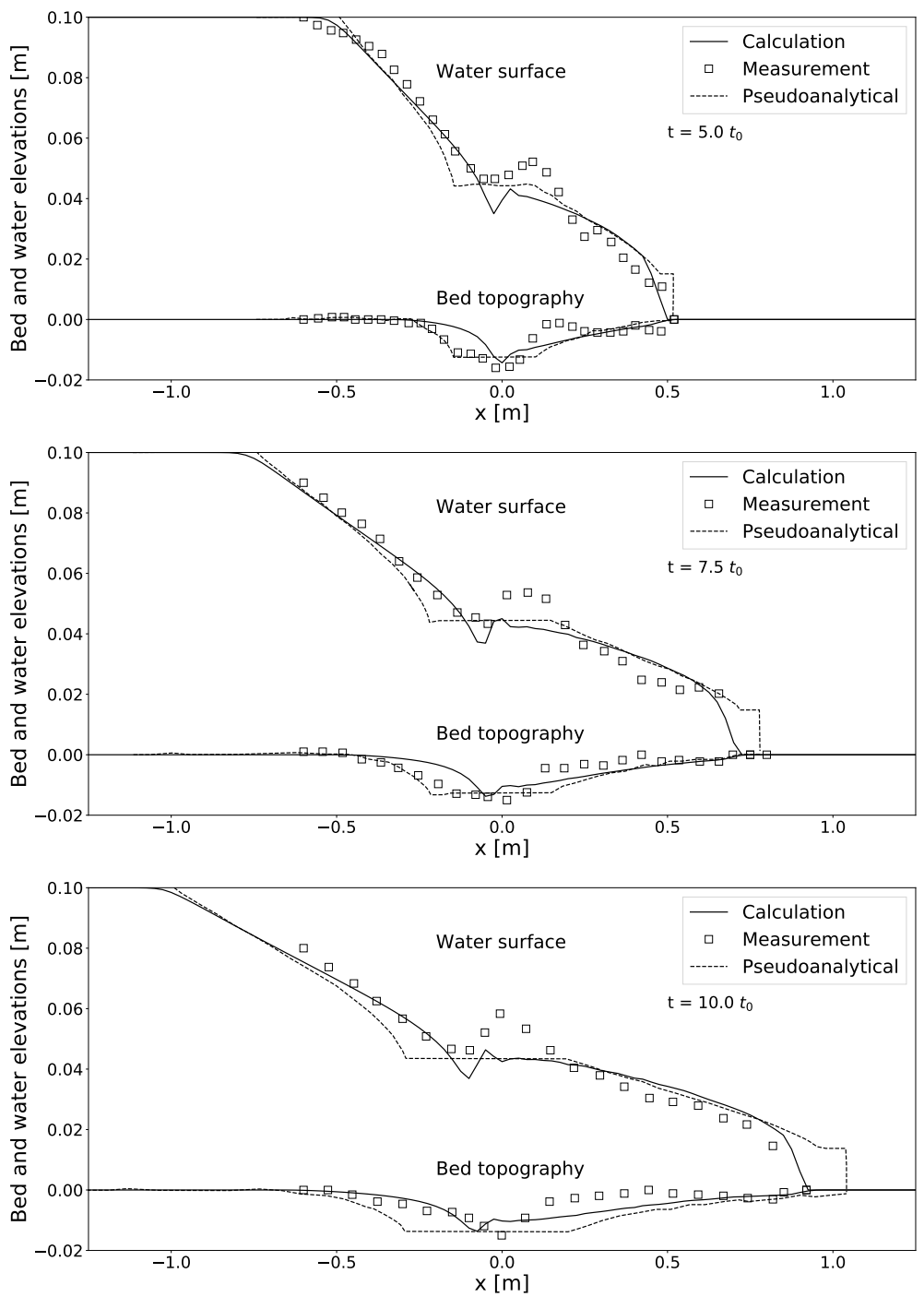

Figure 7: One-dimensional dam-break over movable bed: bed and water surface at: (a) $\mathrm{t}=5.0 \mathrm{t}_{0}$, (b) $\mathrm{t}=7.5 \mathrm{t}_{0}$, (c) $\mathrm{t}=10.0 \mathrm{t}_{0}, \mathrm{t}_{0}=0.101$ S. 

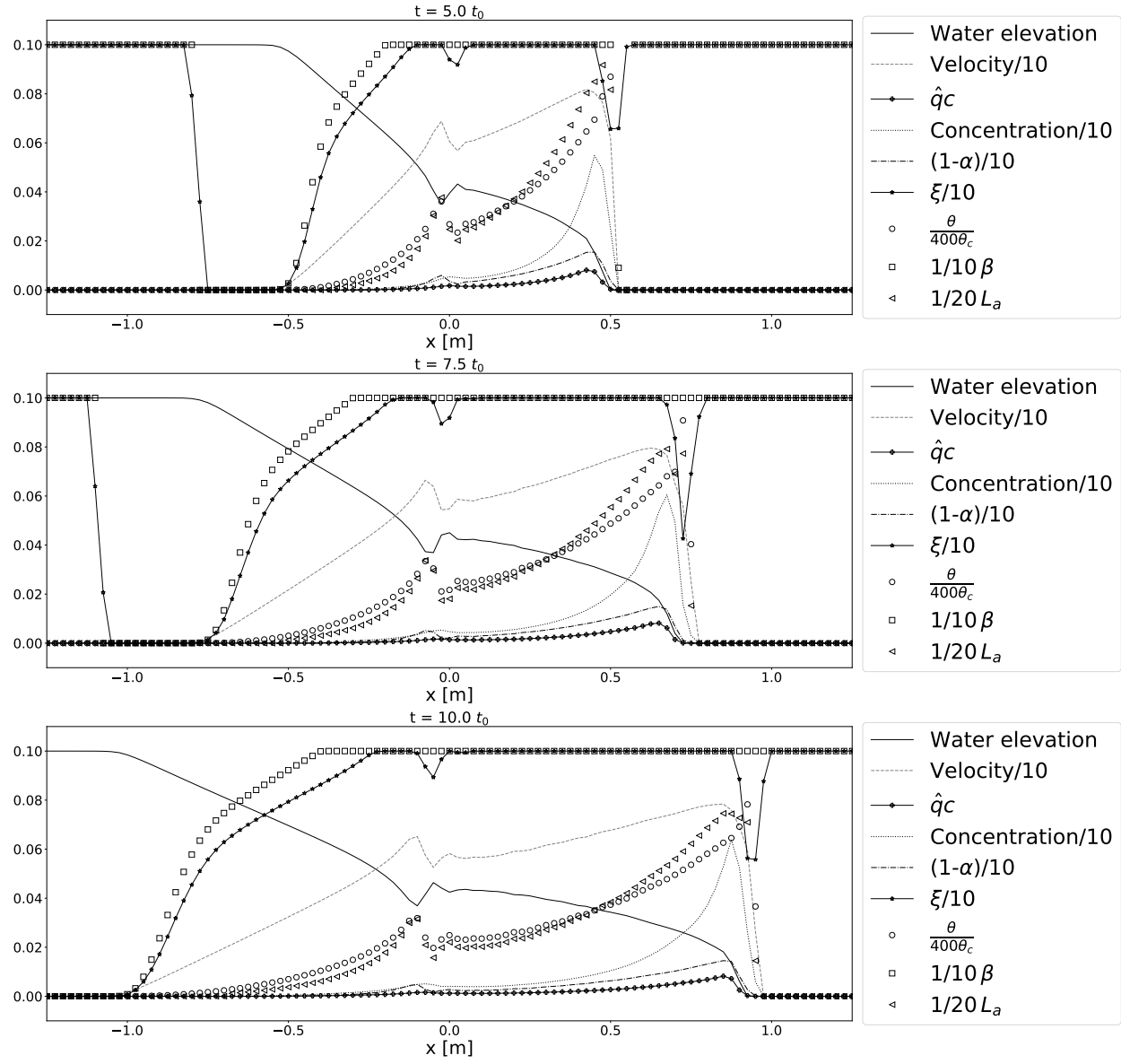

Figure 8: One-dimensional dam-break over movable bed: water level and coefficients along the channel: (a) $t=5.0 t_{0},(b) t=7.5 t_{0},(c) t=10.0$ $\mathrm{t}_{0}, \mathrm{t}_{0}=0.101 \mathrm{~s}$. 
on the bed load velocity coefficient $1 / \beta$ (see Eq. (16)). As described in Sec. 4.1 , the velocity coefficient $\xi$ shows the increasing relationship with the ratio of suspended load. Using a similar manipulation, it can be derived that the larger bed load velocity coefficient $1 / \beta$ will lead to a larger sediment velocity. Eq. (16) reveals that if $\theta / \theta_{c}>20,1 / \beta$ equals 1 and the advection velocity of the sediment is equal to the flow velocity. Fig. 8 shows that $\theta / \theta_{c}$ is located in the range of $[0,40)$, remaining mostly below 20 , while the bed load velocity $1 / \beta$ still reaches 1 . As $u_{*} / u=n \sqrt{g} / h^{1 / 6}$, we can use Eq. (16) to derive that $1 / \beta$ is also influenced by the water depth, and therefore Eq. (16) should be limited as $1 / \beta=\min (1,1 / \beta)$.

\subsubsection{Sensitivity analysis of dam-break over movable bed}

In order to investigate the influence from the different parameters, and quantity how different parameters outperform for the dam-break flows, the sensitivities of the Menning number $n$, sediment diameter $d$, and sediment porosity $p$ are undertaken in this section.

The open-source Python library SALib [19] is applied here to do a global sensitivity analysis. A group of parameters generated by the algorithms from [38] the range of parameters is set to be $\left[0.5 n_{0}, 1.5 n_{0}\right]$, $\left[0.5 d_{0}, 1.5 d_{0}\right]$, and $\left[0.5 p_{0}, 1.5 p_{0}\right]$, which the subscript 0 means the parameters used in the Sec. 4.2.1. The Sobol's sensitivity analysis is performed based on the results from 80 times simulations, the evaluation of the computational model is calculated via Eq. (72) at time $t=7.5 t_{0}$, the results from Sec. 4.2.1 are chosen as the benchmark results.

The first-order sensitivity indices (S1) and the total-order sensitivity index (ST) of the parameters are shown in Tab. 1. The first-order 
Table 1: Results of sensitivity analysis

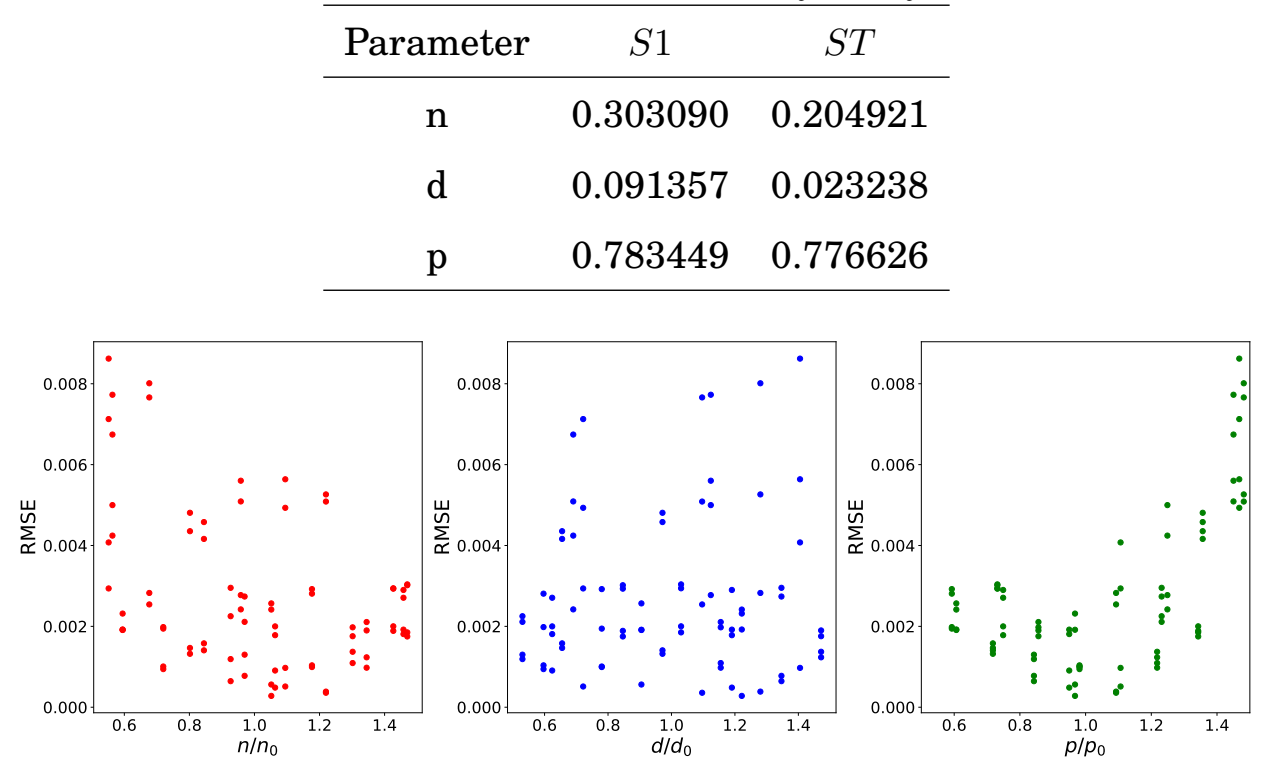

Figure 9: One-dimensional dam-break over movable bed: relationship between the parameters' relative value and RMSE.

sensitivity indices ( $\mathrm{S} 1$ ) shows that the porosity $p$ give the most sensitivity in this numerical model, and sediment diameter $d$ provide the least sensitivity, the total-order sensitivity index shows that the porosity $p$ receives the least sensitivity by the interactions from the other parameters. The relationship between the parameters' relative value and the RMSE can be seen in Fig. 9.

The linear increased parameters are chosen for evaluating the sensitivity from the single parameter, the parameter are set into five levels (e.g. $\left.n / n_{0}=0.5,0.75,1.0,1.25,1.5\right)$ compare to the value set in Sec. 4.2.1. The water surface and bed elevation at time $t=7.5 t_{0}$ are shown in the left side of Fig. 10, it can be observed that the sediment diameter 
$d$ shows very slight influence for the water surface, bottom elevation, and the discharge, which is match the global sensitivity analysis; the Manning number $n$ shows highly influences the discharge, the speed of wave front in the downstream give a linear decrease with the increasing of value $n$, but the shape of the position of maximum erosion hole and the secondary shock at the middle is quite similar; the porosity $p$ of the bed shows more influence on the topography of the bed, even the shock wave front shows the different velocity for the different porosity, but the distribution of the discharge in the downstream is similar, while with the increasing of porosity $p$, the the position of maximum erosion hole and the secondary shock at the middle is moving to the upstream direction and the erosion depth is getting bigger, which also explain why the porosity $p$ give the most sensitivity in the global sensitivity analysis when the evaluation is calculated based on the influence on the bottom elevations.

\subsection{Dyke erosion due to flow overtopping}

Flow overtopping of dykes can cause serious erosion and even wash out structures. Such a complex process is involving outburst, supercritical and steady flow making the simulation of sediment movement even more difficult. Aim of this example is to test the proposed model for each complex flow condition and the influence of different slope effects on the sediment movement.

The laboratory experiment from [47] is replicated numerically. The experimental set-up is sketched in Fig. 11. The flume is $35 \mathrm{~m}$ long and $1 \mathrm{~m}$ wide. The dyke is $0.8 \mathrm{~m}$ high and $1 \mathrm{~m}$ wide, and is located at the mid- 

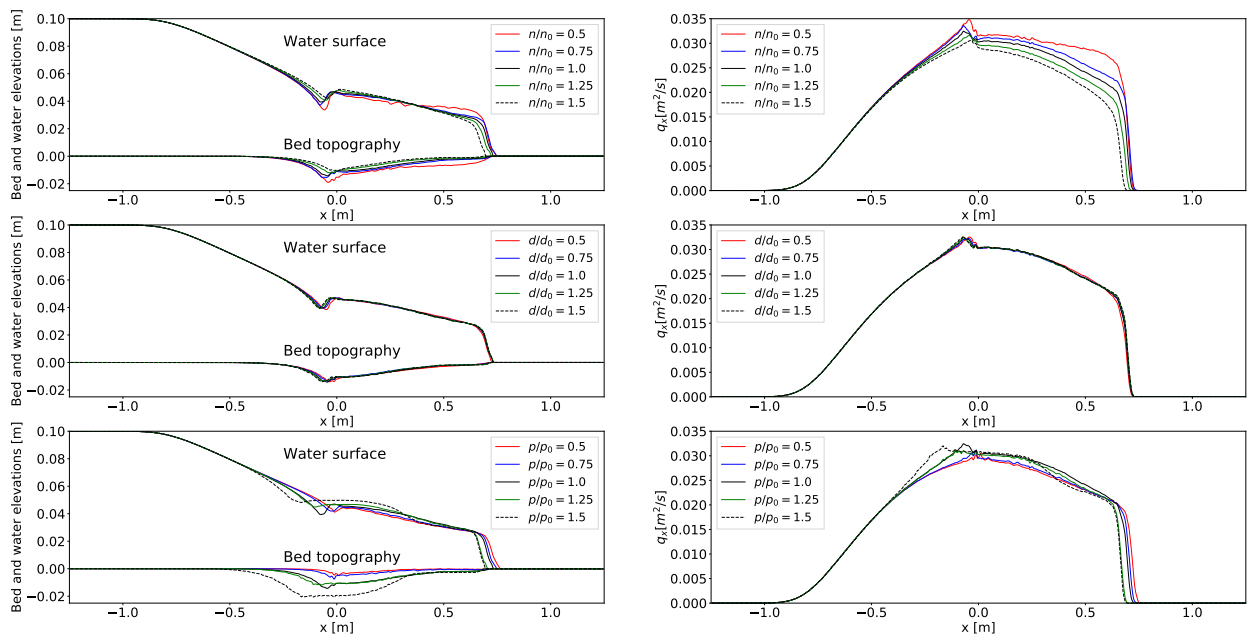

Figure 10: One-dimensional dam-break over movable bed: water surface and bed elevation change with the increasing of parameters (left) and the corresponding discharge along $x$ - direction $q_{x}$ (right) at $t=7.5 t_{0}$. 


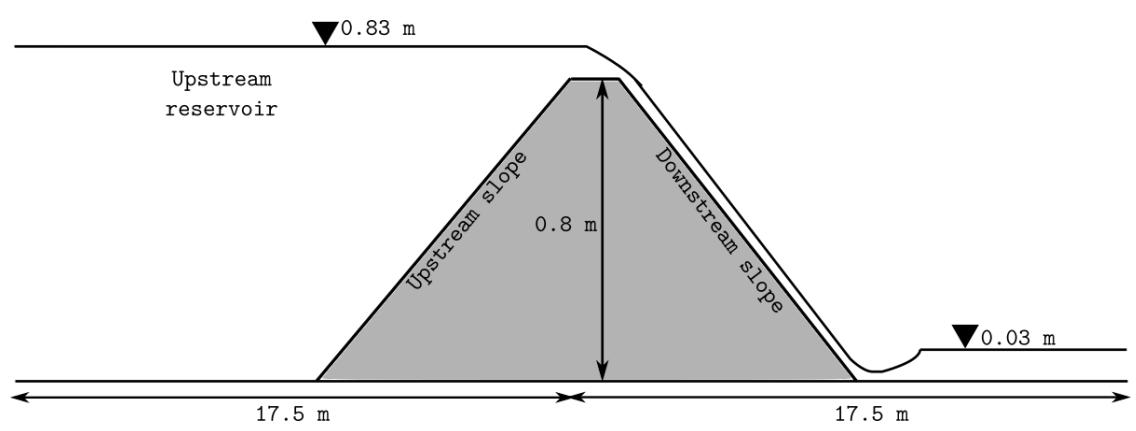

Figure 11: Sketch of overtopping flow over a dyke

dle of the flume with a crest width of $0.3 \mathrm{~m}$. The upstream and downstream slopes of the dyke are $1: 3$ and $1: 2.5$, respectively. The bottom of up- and down-stream of the dyke is fixed and unmovable, the dyke is made of medium sand with a diameter of $d=0.00086 \mathrm{~m}$, and the density of the sand $\rho_{s}=2650 \mathrm{~kg} / \mathrm{m}^{3}$, the porosity of the bed material $p=0.35$, the Manning coefficient is set to $n=0.018 \mathrm{sm}^{-1 / 3}$, the repose angle $\varphi_{r}=26^{\circ}$ and the calibration parameter $\varepsilon=1.2$ after calibration. Initial conditions can be seen via the sketch of the experiment in Fig. 11, a constant water level of $0.83 \mathrm{~m}$ is set at upstream reservoir of the dyke, and $0.03 \mathrm{~m}$ downstream, bottom elevation is $0.0 \mathrm{~m}$ except the dyke, which the downstream slope is initially set to dry. The upstream boundary condition is an inflow boundary, where a constant discharge of $1.23 \cdot 10^{-3} \mathrm{~m}^{3} / \mathrm{s}$ is imposed. The downstream boundary condition is a free outflow condition. The domain is discretized with 1190 triangular cells.

We use the measurement data from the case C-2. The comparison of measured and model predicted bed profiles at $30 \mathrm{~s}$ and $60 \mathrm{~s}$ is shown in Fig. 12 (a-b). The agreement at $30 \mathrm{~s}$ between the simulation results and 

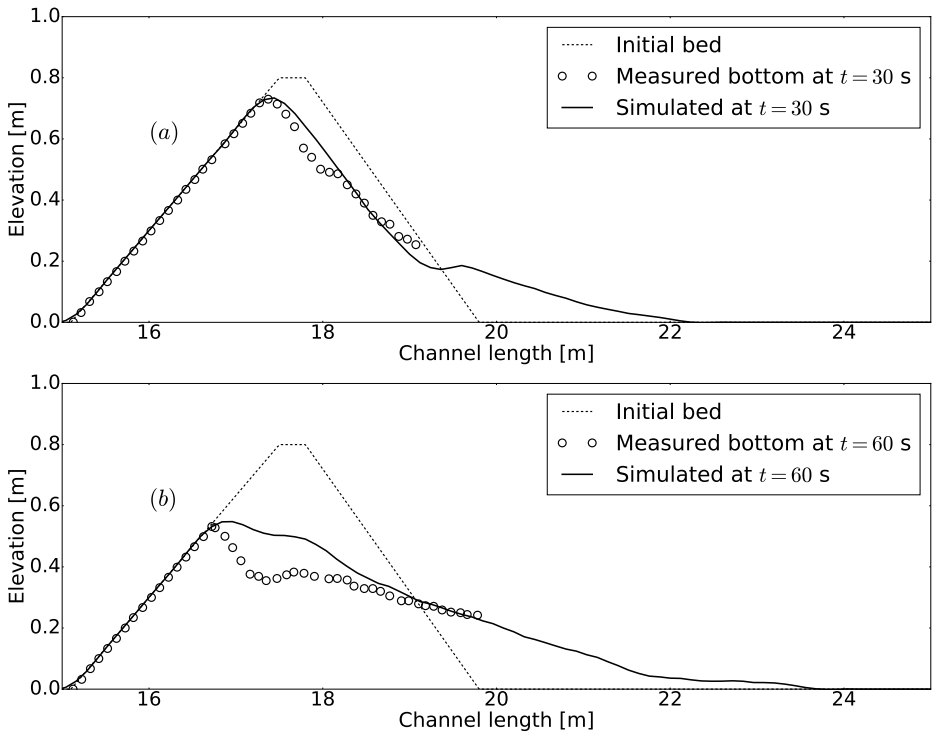

Figure 12: Comparison between simulated bed elevation and measured data at $t=30 \mathrm{~s}(\mathrm{a})$ and $t=60 \mathrm{~s}(\mathrm{~b})$.

the measurement data is fairly good, while it is slightly underestimate the measured erosion at $60 \mathrm{~s}$, there is an obvious scour pit at the peak of the dyke in the observation that is missing in the model prediction.

In addition to measurement data, model results obtained with the SWE-Exner model from [35] and the total load model from [16] (Guan's model hereinafter) are compared with the proposed model. Fig. 13 (a) shows that the proposed model captures the peak in the discharge accurately, but undershoots the measurement data in the later stages of the simulation. We note that the other two models can not replicate this part of the hydrograph neither and the proposed model outperforms both of them. Fig. 13 (b) compares the water elevations. We see that 

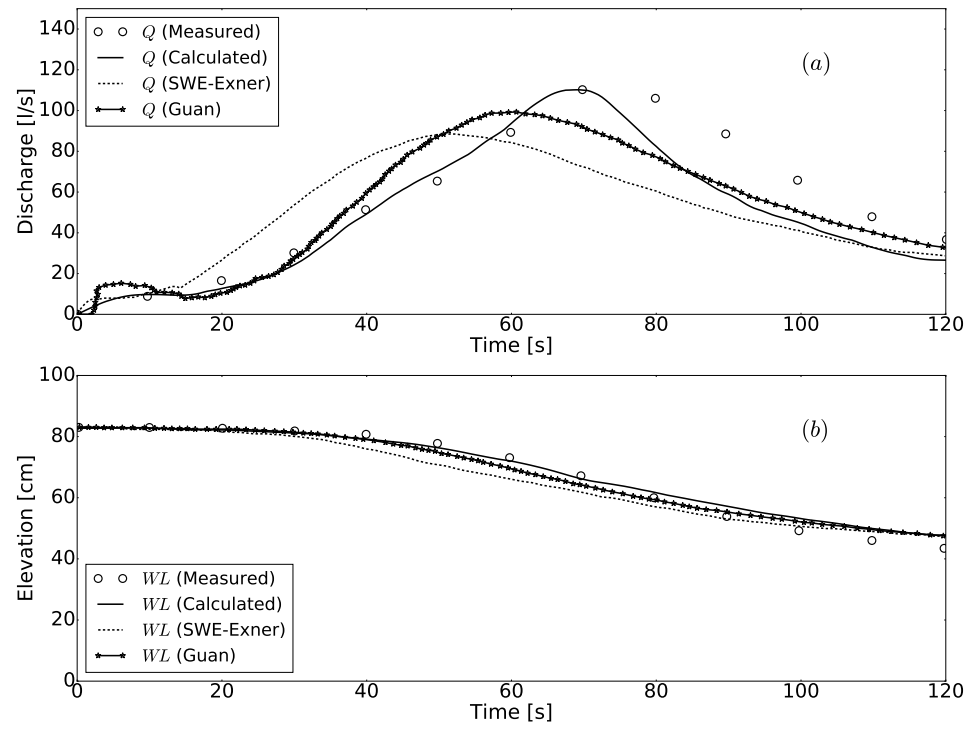

Figure 13: Simulated discharge (a) and water elevation (b) against time compared to the measurement data, SWE-Exner and Guan's model.

water elevations are well predicted for the first $60 \mathrm{~s}$, but overshoot the measurement data after $80 \mathrm{~s}$. This might be due to the effect of the slope on the critical Shield's number $\theta_{c}$ (see Eq. (9), (11), (10)) that influences the erosion on the dyke and the water elevation. Another reason might be the underlying empirical equations that have been derived under different conditions than the investigated case.

Fig. 14 compares different slope effects from Damgaard et al. [9] and Smart and Jäggi [41] that relate to the critical shear stress as seen in Eq. (11) and Eq. (10), respectively. It is seen that the peak discharge from [9] is predicted earlier and lower than [41]. We can conclude that the slope effect significantly influences the flow pattern but has only 
small influence on the water elevation. This means that the erosion at the top of the crest is small, because the critical shear stress of the slope effect is only suitable for a range of bed slope angles and is not valid for this type of topography. We investigate the sensitivity of the slope effect for different values of the repose angle $\varphi_{r}: 26^{\circ}, 30^{\circ}, 35^{\circ}$ and $40^{\circ}$. The model results obtained with these angles are plotted in Fig. 15 and 16. We see that the peak of the discharge shifts to an earlier point in time as $\varphi_{r}$ increases. The maximum discharge decreases for larger values of $\varphi_{r}$. Meanwhile, larger $\varphi_{r}$ values lead to higher water elevations at the upstream. This can be explained by the increased critical shear stress on the slope, which is proportional to $\varphi_{r}$ as seen in Eq. (11) and 10.

Parameters include suspended transport ratio $1-\alpha$ (see Eq. (14)), sediment velocity coefficient $\xi$ (see Eq. (4)) and the slow velocity $u$ which used for controlling the sediment transport mode are presented in Fig. 17. The relationship between the parameters is similar to what has been discussed in Sec. 4.2. By comparing $(1-\alpha)$, we can argue that the results of the proposed scheme are influenced more significantly by the bed load transport, while the results obtained from [16] are more significantly influenced by the suspended load transport. Eq. (14) reveals that the sediment settling velocity $\omega_{s}$ is the parameter that indicates which transport mode is more significant. In this work, we calculate $\omega_{s}$ via Eq. (12), while [16] treats $\omega_{s}$ as a calibration parameter. This explains the difference in the results. 

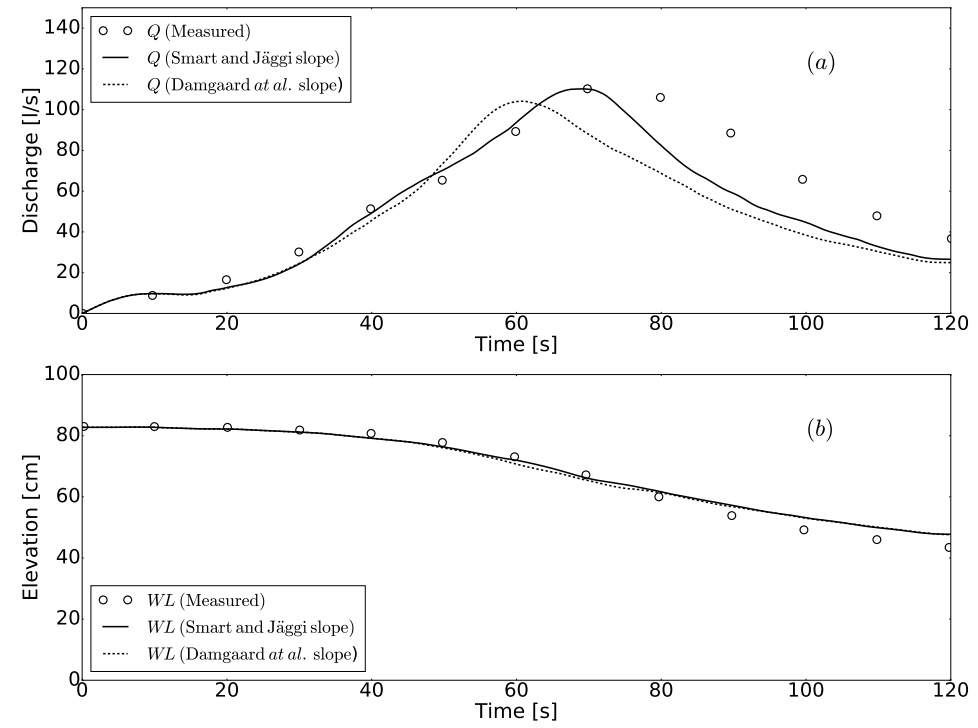

Figure 14: Comparison of measurement data with slope effect from Smart and Jäggi [41] and Damgaard et al. [9] for simulated discharge (a) and water elevation (b) against time. 

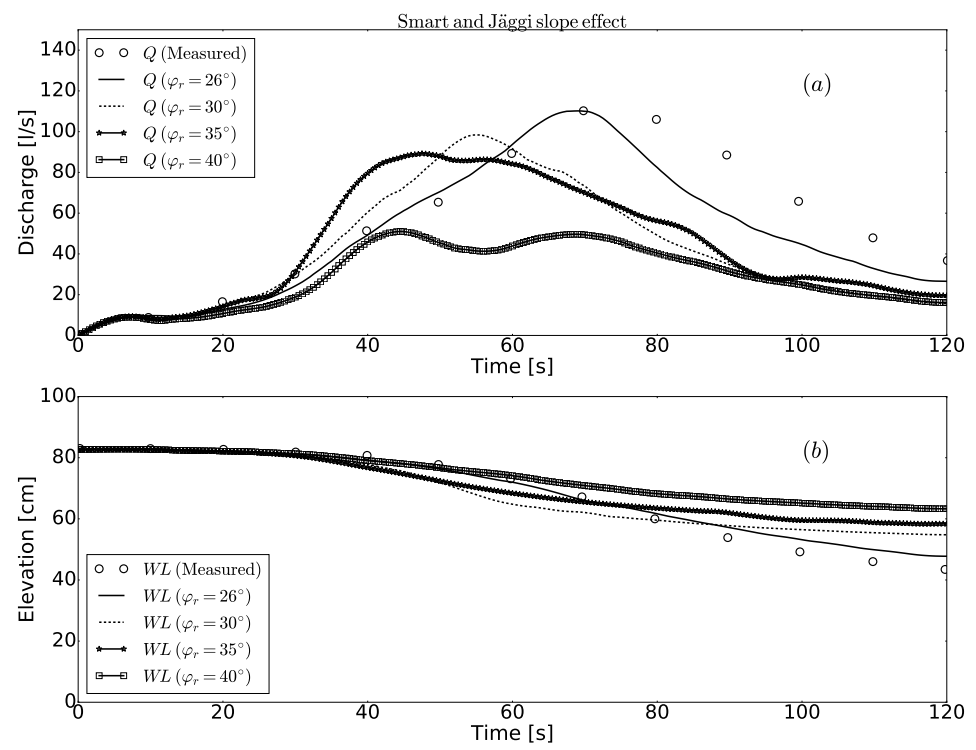

Figure 15: Comparison of measurement data with slope effect from Smart and Jäggi [41] for different repose angle $\varphi_{r}$ for simulated discharge (a) and water elevation (b) against time. 

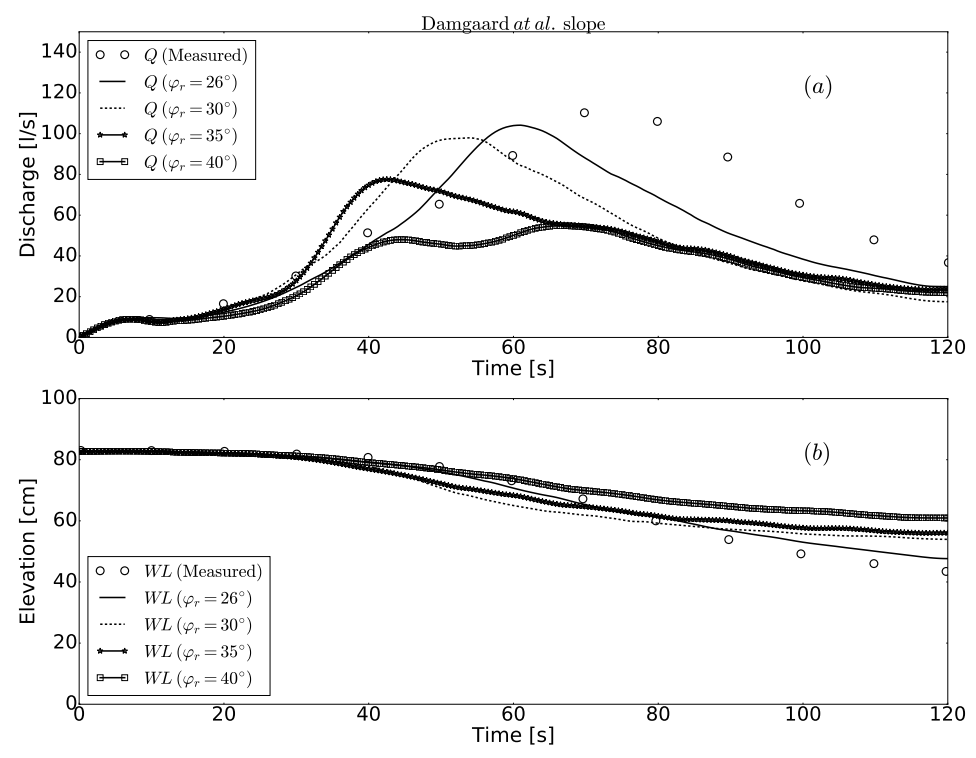

Figure 16: Comparison of measurement data with slope effect from Damgaard et al. [9] for different repose angle $\varphi_{r}$ for simulated discharge (a) and water elevation (b) against time. 

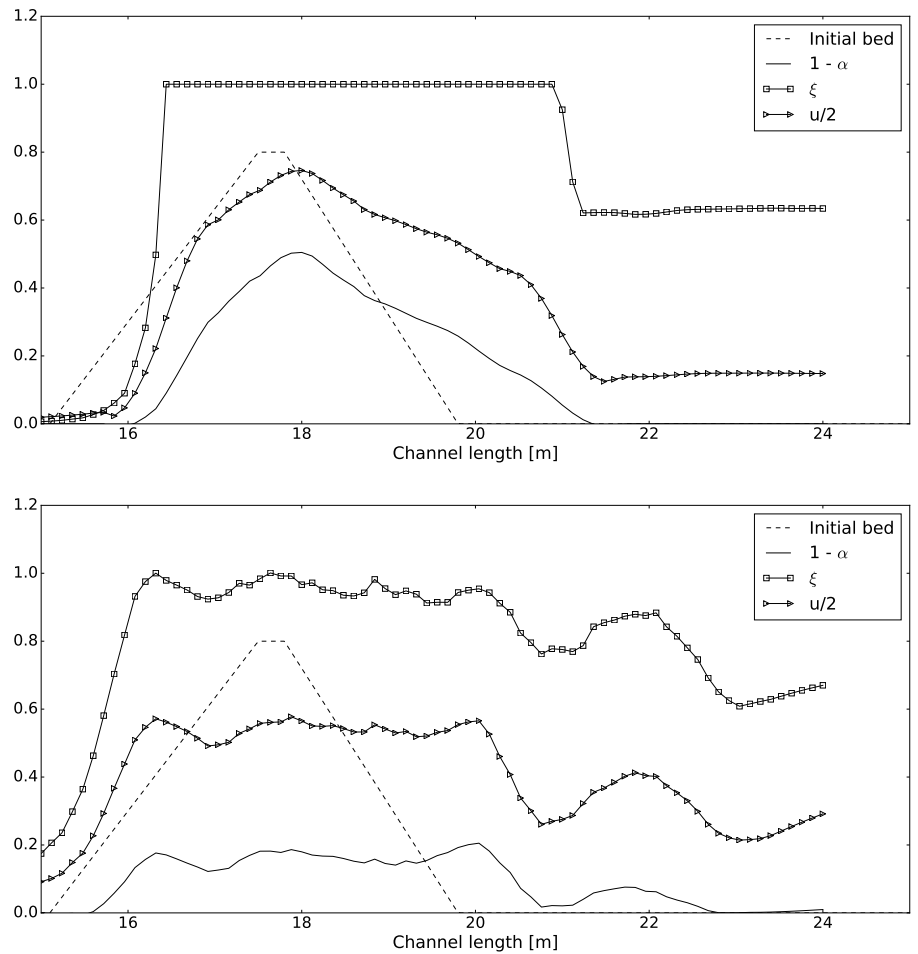

Figure 17: Simulated coefficients at $t=30 \mathrm{~s}$ and $t=60 \mathrm{~s}$. 


\subsection{Two-dimensional dam-break flow in a mobile channel with a sudden enlargement}

In this test case, we aim to assess the suitability of the proposed scheme to two-dimensional problems. The laboratory experiment described in [13] is reproduced numerically. The flume in the experiment is $6 \mathrm{~m}$ long and features a sudden enlargement from $0.25 \mathrm{~m}$ to $0.5 \mathrm{~m}$ width, which is located at $1 \mathrm{~m}$ downstream of the gate, cf. Fig. 18. The initial conditions consisted of a $0.100 \mathrm{~m}$ horizontal layer of fully saturated and compacted sand over the whole flume and an initial layer of $h_{0}=0.25 \mathrm{~m}$ clear water upstream of the gate water depth at the upstream of the gate and dry bed in the downstream. The median sediment diameter is $d=1.65 \mathrm{~mm}$, the density is $\rho_{s}=2630 \mathrm{~kg} / \mathrm{m}^{3}$, the repose angle $\varphi=30^{\circ}$ and the porosity of the sand is $p=0.42$. Bed friction is accounted for via a Manning's coefficient of $n=0.0185 \mathrm{sm}^{-1 / 3}$. At the beginning of the experiment, the gate is opened to generate a dam break wave. In the numerical model, we use 2064 triangular cells to discretize the flume. The calibration parameter is determined to be $\varepsilon=0.15$ in this test case. Measurement data of water and bed elevations at specific gauges and cut sections are available from [13], cf. Tab. 2 and 3, respectively. The three dimensional results from a standard $k-\epsilon$ model (3D results) obtained from [33] are chosen here for comparison.

Fig. 19 shows the comparison of measured and computed water elevations. We see that overall the model prediction is fairly close to the measurement data. Gauges U1 and U3 show the worst agreement. Especially for $\mathrm{U} 1$, the $3 \mathrm{D}$ results almost perfectly match the measurement data, but for results from this work overestimate the water level. Sim- 
Table 2: Position of gauges

\begin{tabular}{ccc} 
Gauge & $x(\mathrm{~m})$ & $y(\mathrm{~m})$ \\
\hline $\mathrm{U} 1$ & 3.75 & 0.125 \\
$\mathrm{U} 2$ & 4.20 & 0.375 \\
$\mathrm{U} 3$ & 4.20 & 0.125 \\
$\mathrm{U} 4$ & 4.70 & 0.375 \\
$\mathrm{U} 5$ & 4.70 & 0.125 \\
\hline
\end{tabular}

Table 3: Position of cut sections

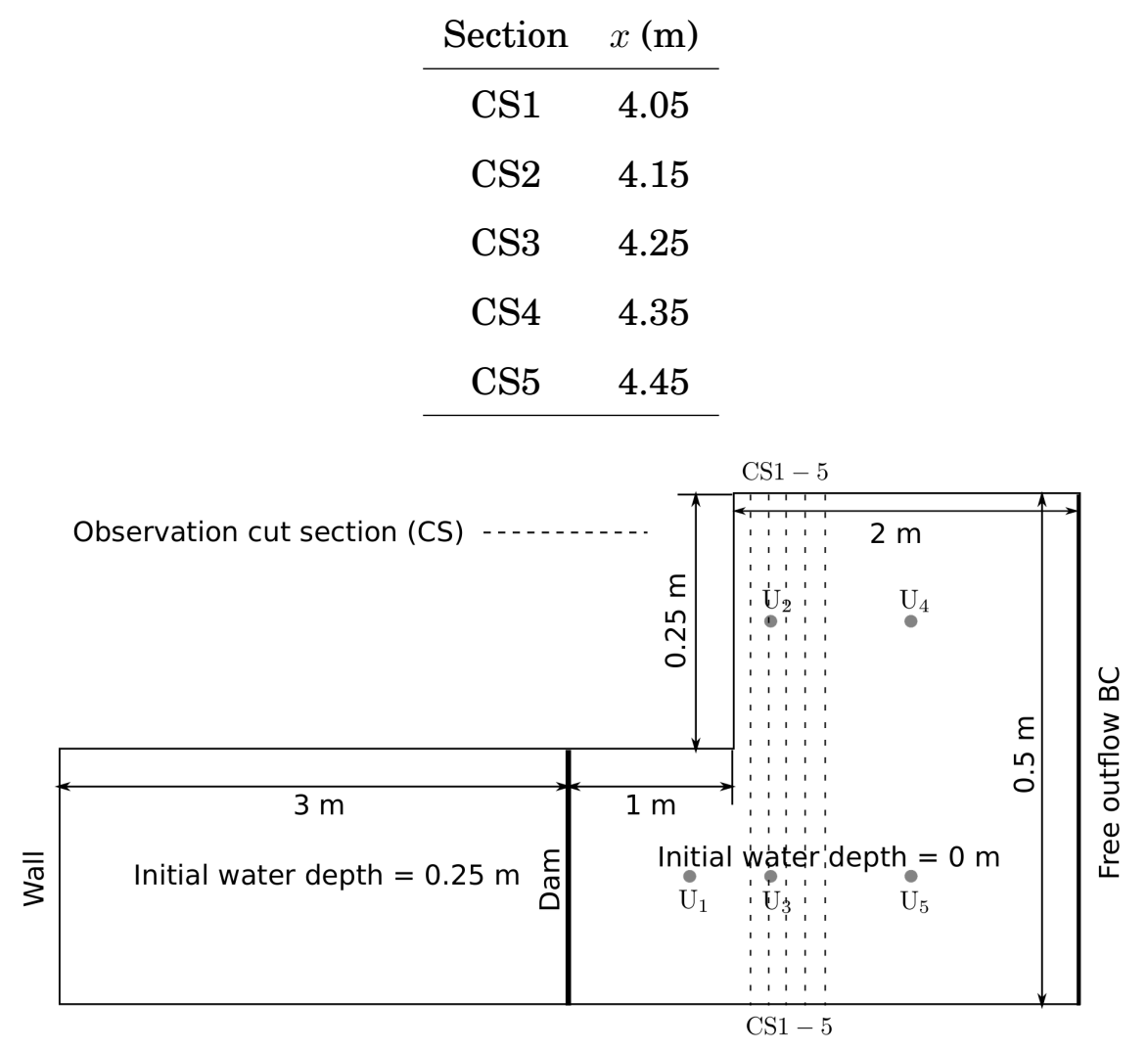

Figure 18: Sketch of a 2D dam-break flow with a sudden enlargement channel over mobile bed. 
ilarly, for the results at $\mathrm{U} 3$, both the results from 3D model and this work underestimate the measurement, but the $3 \mathrm{D}$ results show slightly better agreement. The reason for the deviation is that these gauges are located close to the expansion where strongly three-dimensional flow occurs. The depth-averaged model concept is poor at these locations. While, at U2, the results from this work show slightly better agreement than the 3D model results, both models provide good results at the remaining gauges. This supports the conclusion that the deviation at U1 and $\mathrm{U} 3$ are due to strong 3D effects at these locations.

Fig. 20 shows the comparison between measured and computed bed elevations at cut sections CS1 to CS5, at the end of the simulation. We see that all cut sections are predicted reasonably well by the numerical model. The overall tendency of erosion on the right side and deposition on the left side of channel is captured accurately. At CS1, which is located close to the expansion area, the maximum erosion is underestimated and its location is predicted wrong, more specifically it is shifted to the left, while the $3 \mathrm{D}$ results almost perfectly capture the magnitude of maximum erosion and its location, the deposition at the left bank is predicted wrong with an erosion hole instead. At CS2 to CS5, deviations between the measured and predicted maximum erosion is observed. The maximum deposition locations are predicted more accurately in $3 \mathrm{D}$ results. A consistent shift to left of the maximum deposition locations in the simulation results from this work can be observed. Three-dimensional flow effects are most likely the reason for these deviations. The proposed model is depth-averaged, and therefore neglects three-dimensional effects. This means that there will be more flow predicted into the down-stream direction of the channel, which might be 

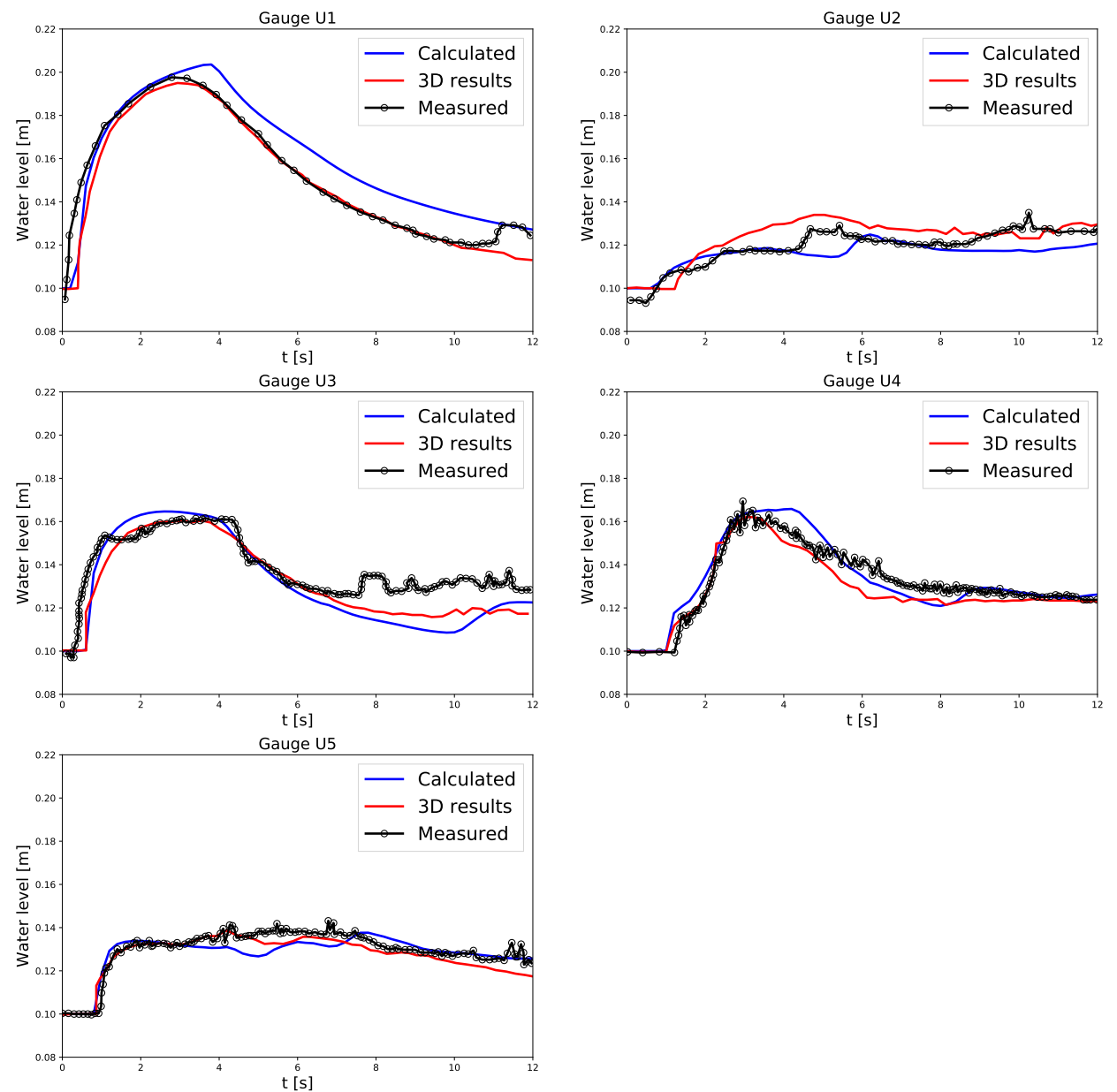

Figure 19: Comparison between measured (-o-) and calculated (-) water levels at gauges U1-U6. 
the reason for more erosion at the right side and less deposition at the left side. We show the computed final bed elevation contours in Fig. 21.

\subsection{Partial dam-break flow on movable bed in a straight channel}

In this final example, we test the proposed model again for complex twodimensional flow conditions, the computational domain is a suddenly enlarged channel with symmetric geometry. As the proposed model is discretizated on the unstructured grids, the complex geometry conditions can be thought as a good benchmark for verifying the sediment movement and whether the flow field is influenced by the sediment interaction which leads to a non-symmetric flow field. The laboratory experiment from $[42,58]$ is reproduced numerically. The flume is $3.6 \mathrm{~m}$ wide and $36 \mathrm{~m}$ long, cf. Fig. 22. A $1 \mathrm{~m}$ wide gate is located in the middle of the domain, the partial dam-break was represented by rapidly lifting the gate away. Initially, a sand layer with a depth of $85 \mathrm{~mm}$ is set over a fixed bed in the region that spans from $1 \mathrm{~m}$ upstream of the gate to $9 \mathrm{~m}$ downstream of the gate and is indicated with gray color in Fig. 22. The density of the sand layer is $\rho_{s}=2630 \mathrm{~kg} / \mathrm{m}^{3}$ and its porosity is $p=0.42$. The diameter of the sediment is $d=0.00161 \mathrm{~m}$, and the repose angle $\varphi_{r}=30^{\circ}$. The origin of the coordinate system is located at the middle of the gate. Water and bed elevations are measured at 8 gauges. Gauges 1-4 are located at the coordinates $x=0.64 \mathrm{~m}$ with $y_{1}=-0.5, y_{2}=-0.165, y_{3}=0.165, y_{4}=0.5 \mathrm{~m}$, respectively, gauges 5-8 are located at the coordinates $x=1.944 \mathrm{~m}$ with $y_{5}=-0.99, y_{6}=$ $-0.33, y_{7}=0.33, y_{8}=0.99 \mathrm{~m}$, respectively. Three longitudinal cut sec- 

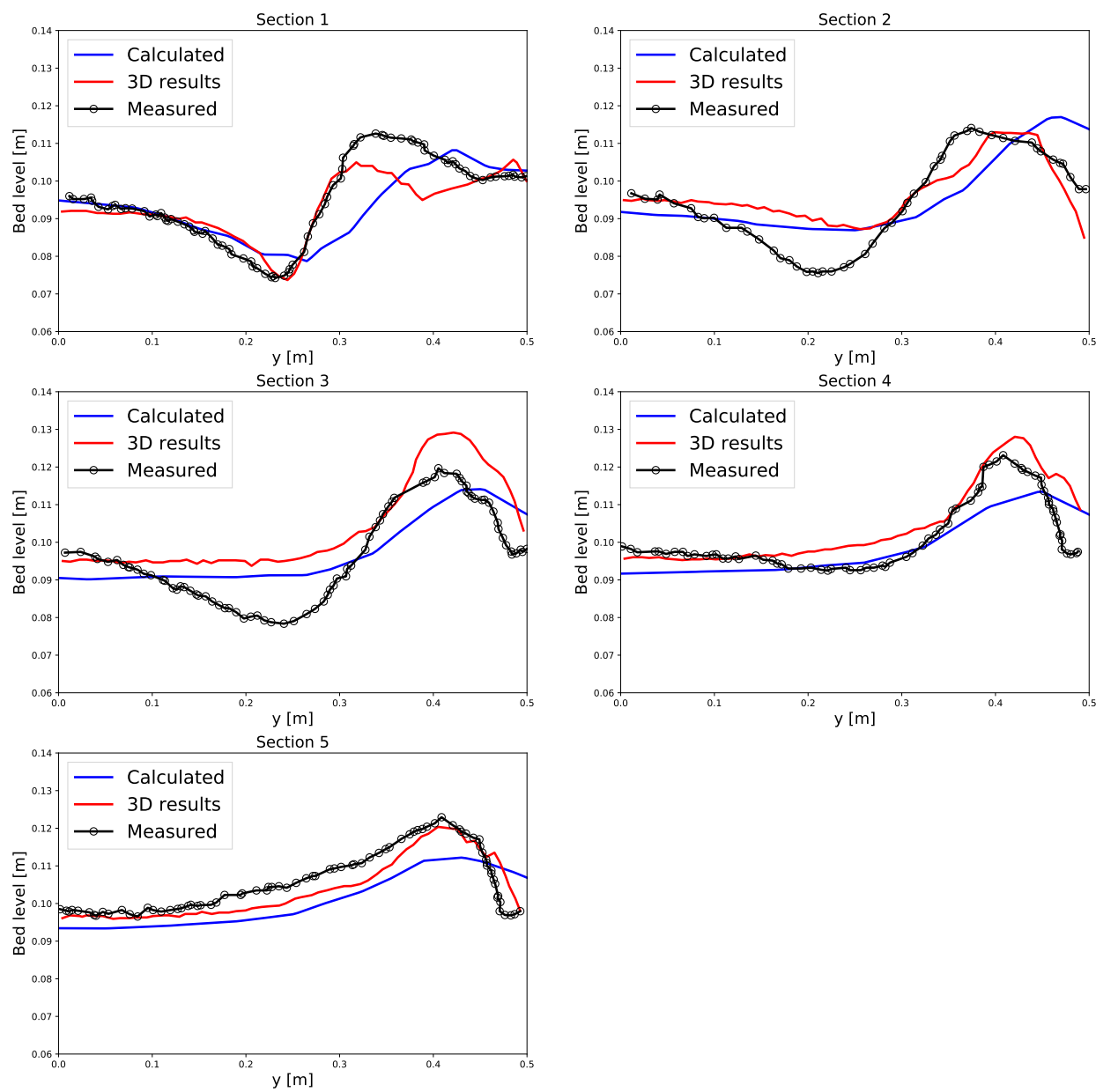

Figure 20: Comparison between measured (-o-) and calculated (-) bottom topographies at cut sections CS1-CS5. 


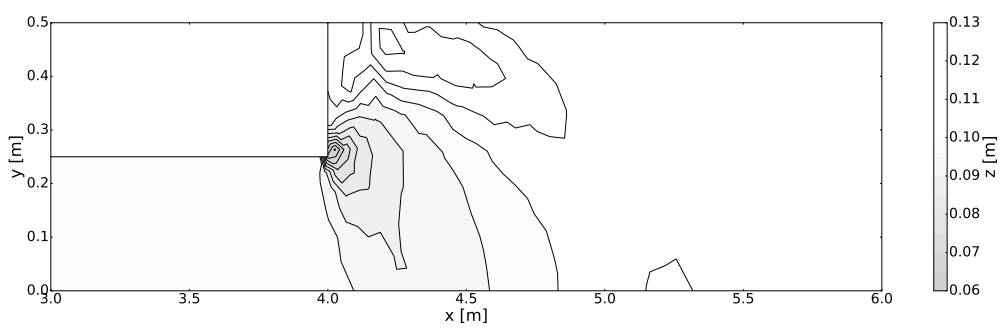

Figure 21: Contour plot of calculated final bed topography.

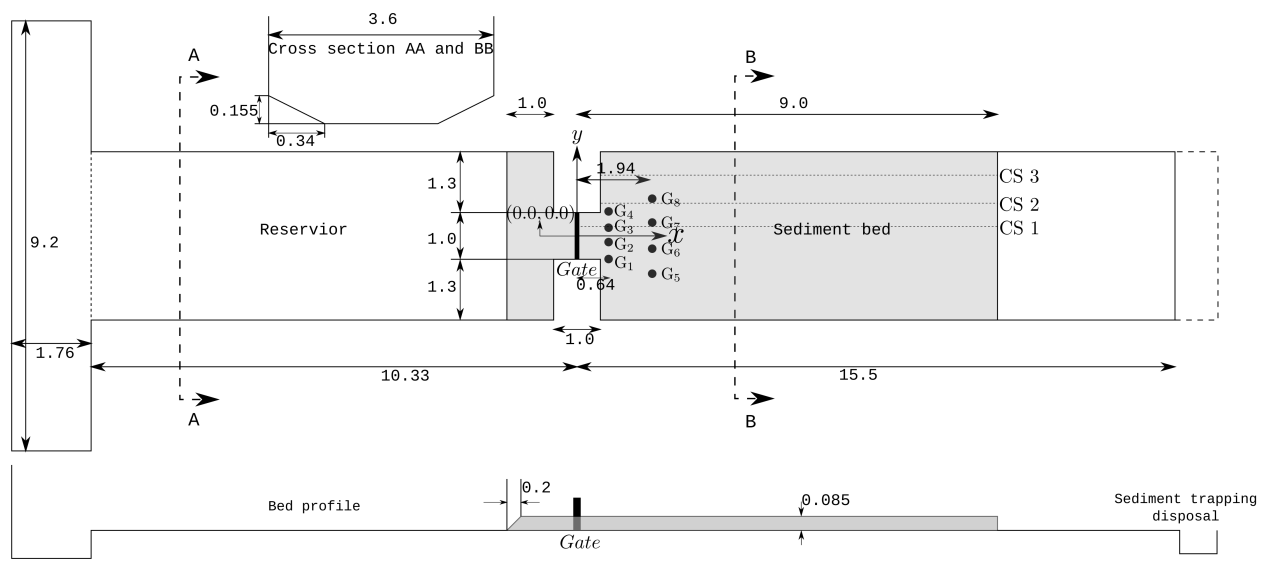

Figure 22: Sketch of UCL partial dam-break experiment (dimension in meters) after [58]

tions are chosen to measure the final bed topography, all the cut sections are set along the $x$ - direction by the range of $[0.0,9.0] \mathrm{m}$, with parallel lines for cut section CS1 to CS3 located at $y=0.2 \mathrm{~m}, y=0.7 \mathrm{~m}$ and $y=1.455 \mathrm{~m}$, respectively, cf. Fig 22 .

The laboratory experiment is repeated twice, i.e. two measurement data sets are available for comparison.

The domain is discretized using 2935 triangular cells. The simulation is run for $20 \mathrm{~s}$. The calibration parameter $\varepsilon=0.75$ is adopted in this test case. The Manning roughness coefficient is $n=0.01 \mathrm{sm}^{-1 / 3}$ for 
the fixed bed, and $n=0.0165 \mathrm{sm}^{-1 / 3}$ for the sand layer [58]. The initial water level in the reservoir is $0.47 \mathrm{~m}$ above the fixed bed, and the dry bed for the downstream. Transmissive boundary conditions are set at the downstream boundary and free slip boundary conditions are set for all other boundaries.

Fig. 23 shows the comparison of measured and computed water elevations at the 8 gauges. We note that the locations of the gauges are symmetric with regard to the $y$-axis. Thus, we observe that the flow is symmetric by comparing the corresponding gauge pairs, i.e. G1 and G4, G2 and G3, G5 and G8, and G6 and G7. The computed water elevations at gauges G5 to G8 show good agreement with the measurement data. At gauges G1 and G4 the computed water elevations undershoot the measurement data, while at G2 and G3 the measurement data is overshot by the numerical model. This is most likely due to the sudden expansion that causes three-dimensional flow conditions in these locations.

The predicted bed elevations at $20 \mathrm{~s}$ along longitudinal cut sections at CS1-CS3 are compared against measurement data in Fig. 24. We see that the model prediction is good in the upstream part for CS1 and CS2. The deposition at the downstream is under-predicted. The bed elevations at CS3 show good agreement. In the upstream, the deposition is underestimated.

\section{Conclusions}

We present a two-dimensional, well-balanced total load sediment transport model that features following novel aspects: (1) the suspended 

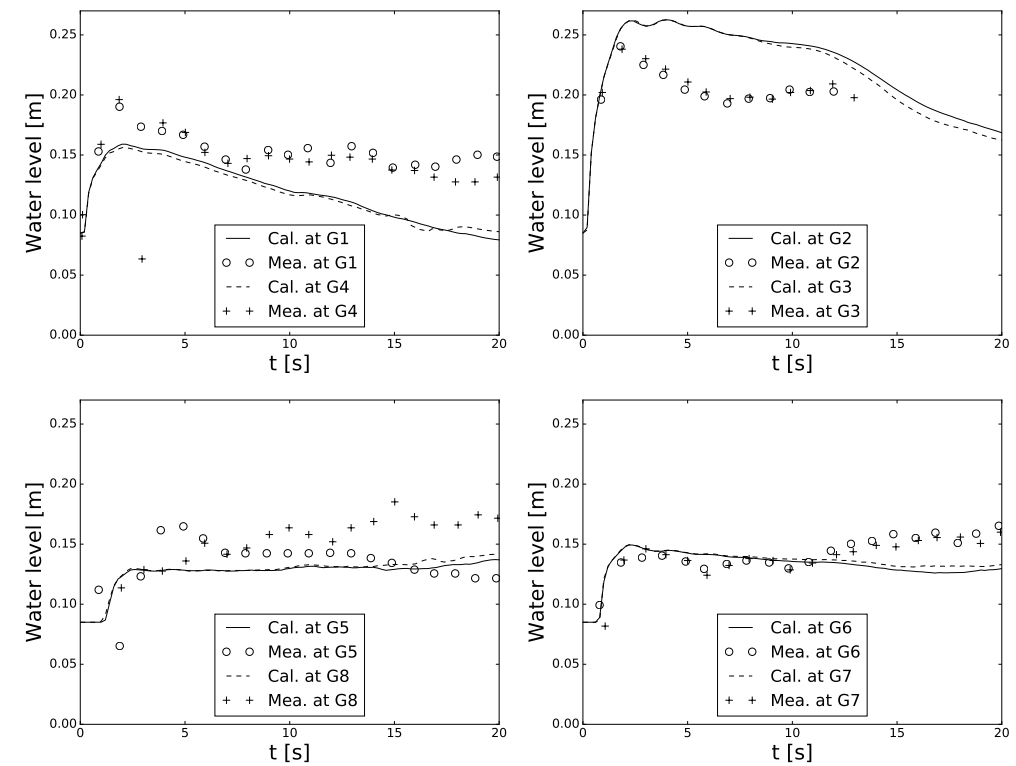

Figure 23: Comparison between measured and calculated water levels at gauges G1-G8. 


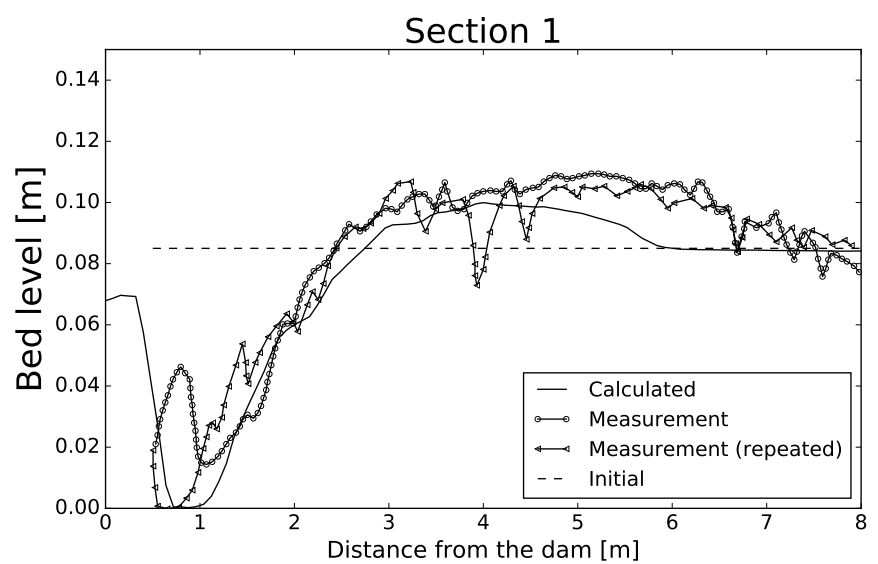

Section 2
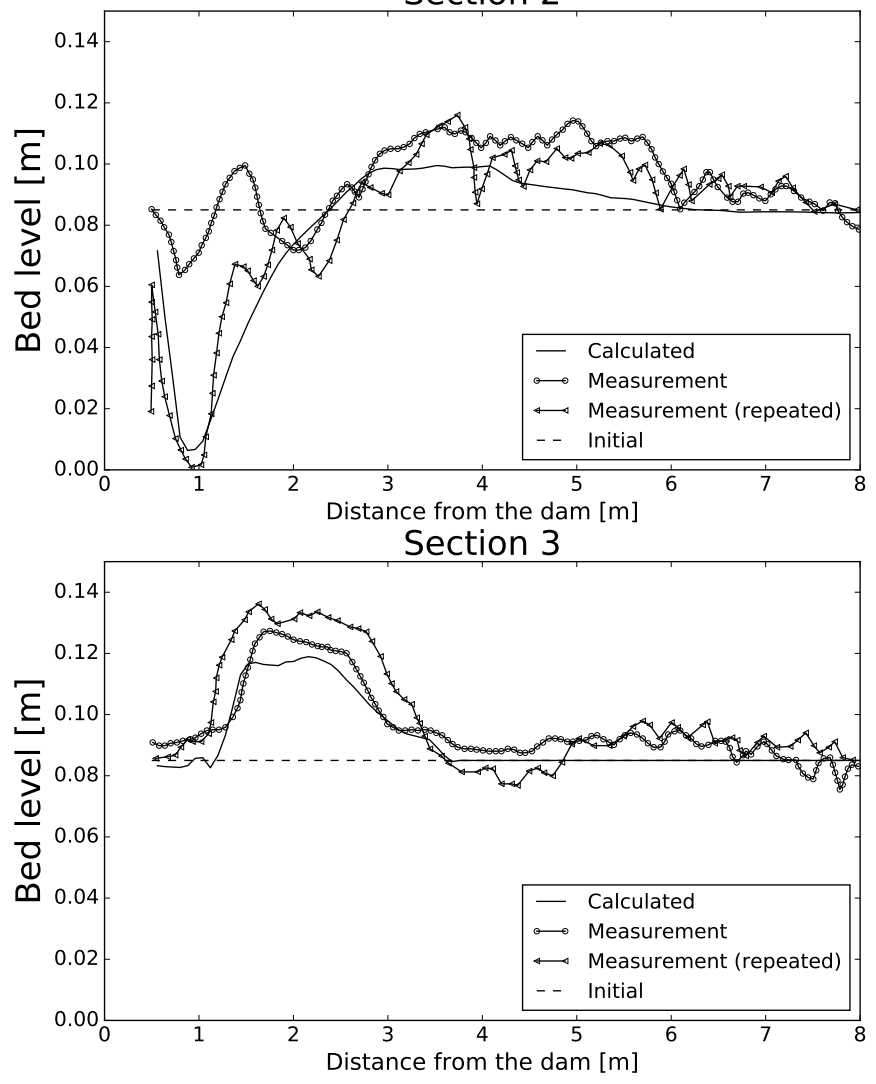

Figure 24: Comparison between measured and calculated bottom topographies at cut sections CS 1,2 and 3 . 
load is advected with a different velocity from that of water, which is achieved by the introduction of the coefficient $\xi$; (2) a novel HLLC approximate Riemann solver is used to take into account the different advection velocities; (3) an improved bed slope treatment that accounts for density variation inside the cell; (4) a novel splitting-point implicit source term discretization for the remaining source terms.

The model is tested in 5 examples that include fixed bed and mobile bed problems. From these examples we can conclude that the hydrodynamic module reproduces the flow fields accurately and the morphodynamic module reproduces the bed evolution fairly well for different types of complex flows such as dyke overtopping, dam-break flow and discontinuous geometry.

A sediment velocity coefficient is introduced to distinguish between flow velocity and sediment advection velocity. This coefficient mainly depends on the ratio of suspended load. The increase of bed load velocity coefficient $1 / \beta$, will lead to a larger sediment advection velocity.

The sediment movement calculation is mainly based on the equation from Meyer, Peter and Müller, which is an empirical equation derived from a group of physical experiments. Situations that satisfy the laboratory conditions are limited. Hence, the validity of the Meyer-Peter and Müller equation for a majority of cases is questionable. The calibration parameter $\varepsilon$ is introduced to account for this issue. Varying this parameter yields a change in the erosion depth, and enables reproducing the measurement data more accurately.

Meanwhile, the slope effect is also found to have a large influence on the sediment movement and the flow pattern during the simulation, as the slope effect will lead to a different critical shear stress number 
$\theta_{c}$, which will lead to a different bed load capacity $q_{b *}$. Hence, the suspended load erosion and the concentration distribution are also influenced. In this work, the slope effect from [41] is found to outperform other formulations, but it must be mentioned that we did not perform tests that consider different initial bed gradients.

A sensitivity analysis is undertaken against the one-dimensional dam-break flow over movable bed, parameters include Manning number $n$, sediment diameter $d$, and sediment porosity $p$ are chosen as the sensitive parameters, the results show that the diameter of sediment $d$ gives the least influence and sensitivity for the numerical model, Manning number $n$ shows more sensitivity for the water discharge, the erosion depth also get influenced but the position of the shock wave in the middle and maximum erosion hole keep doesn't get influenced, the porosity $p$ shows more sensitivity on the erosion hole depth and shape for the sediment, but for the water surface and the discharge in the downstream, the influence is quite small.

On a final note, we discuss some limitations of the model. The proposed model uses depth-averaged approach. Consequently, if threedimensional effects or large horizontal circulation patterns become significant, e.g. turbulent vertical structures and non-hydrostatic pressure distribution, the model's underlying assumptions are violated and model accuracy can not be guaranteed. In the range of classical shallow flow theory, the proposed model is expected to predict the flow field and the sediment movement with reasonable confidence. Depth-averaged models are useful for applications considering large-scale far-field results for real-world cases, where the influence of localized three-dimensional effects can be neglected in the "larger picture". 
The proposed model further assumes non-cohesive sediment. On the other hand, the basic assumption for suspended load theory is that the diameter of the sediment is much smaller than the water mass scale. With this assumption, the velocity of suspended load is thought to be equal to the velocity of the fluid in all horizontal directions. For bed load, the sediment diameter and the water mass scale are almost at the same order of magnitude, and a different transport velocity must be assumed [8]. All of these findings are valid only for cases with relatively low sediment concentration. If the sediment concentration is high, the fluid-sediment mixture will become a non-Newtonian fluid, and all our assumptions would fail. Thus, the proposed model is limited to low sediment concentrations. This limitation is not unique for the proposed model, but also applies to all sediment transport models discussed in the introduction.

While we discussed the limitations of the proposed model, we emphasize that the model is reliable and accurate for a broad range of applications in hydro- and environmental system modeling, and improves existing shallow flow sediment transport models. Future work will aim to extend the range of model's capability, e.g. by using a multi-layer shallow flow model to capture the three dimensional effects, and including turbulence models.

\section{Acknowledgement}

The authors gratefully acknowledge the China Scholarship Council and the TU Berlin, Germany for the scholarships granted to J. Zhao 


\section{References}

[1] A. Armanini and G. Di Silvio. A one-dimensional model for the transport of a sediment mixture in non-equilibrium conditions. Journal of Hydraulic Research, 26(3):275-292, 1988.

[2] E. Audusse and M. O. Bristeau. A well-balanced positivity preserving "second-order" scheme for shallow water flows on unstructured meshes. Journal of Computational Physics, 206:311-333, 2005.

[3] J. P. Bennett. Concepts of mathematical modeling of sediment yield. Water Resources Research, 10(3):485-492, 1974.

[4] A. Bermudez and M. E. Vázquez. Upwind methods for hyperbolic conservation laws with source terms. Computers \& Fluids, 23(8):1049-1071, 1994.

[5] T. Buffard and S. Clain. Monoslope and multislope MUSCL methods for unstructured meshes. Journal of Computational Physics, 229(10):3745-3776, 2010.

[6] T. R. Bussing and E. M. Murman. Finite-volume method for the calculation of compressible chemically reacting flows. AIAA journal, 26(9):1070-1078, 1988.

[7] Z. Cao, G. Pender, S. Wallis, and P. Carling. Computational dambreak hydraulics over erodible sediment bed. Journal of hydraulic engineering, 130(7):689-703, 2004.

[8] N. Chien and Z. Wan. Mechanics of sediment transport. 1999. 
[9] J. S. Damgaard, R. J. Whitehouse, and R. L. Soulsby. Bed-load sediment transport on steep longitudinal slopes. Journal of Hydraulic Engineering, 123(12):1130-1138, 1997.

[10] H. A. Einstein et al. The bed-load function for sediment transportation in open channel flows, volume 1026. Citeseer, 1950.

[11] L. Fraccarollo and H. Capart. Riemann wave description of erosional dam-break flows. Journal of Fluid Mechanics, 461:183-228, 2002.

[12] L. Fraccarollo and E. F. Toro. Experimental and numerical assessment of the shallow water model for two-dimensional dam-break type problems. Journal of hydraulic research, 33(6):843-864, 1995.

[13] L. Goutiere, S. Soares-Frazão, and Y. Zech. Dam-break flow on mobile bed in abruptly widening channel: experimental data. Journal of Hydraulic Research, 49(July 2015):367-371, 2011.

[14] B. Greimann, Y. Lai, and J. Huang. Two-Dimensional Total Sediment Load Model Equations. Journal of Hydraulic Engineering, 134:1142-1146, 2008.

[15] M. Guan, N. G. Wright, F. Asce, and P. A. Sleigh. Multimode Morphodynamic Model for Sediment-Laden Flows and Geomorphic Impacts. Journal of Hydraulic Engineering, 141(6):1-12, 2015.

[16] M. Guan, N. G. Wright, and P. A. Sleigh. 2d process-based morphodynamic model for flooding by noncohesive dyke breach. Journal of Hydraulic Engineering, 140(7):04014022, 2014. 
[17] V. Guinot. Godunov-type Schemes: An introduction for engineers. Elsevier Science B.V., Amsterdam, The Netherlands, 1 edition, 2003.

[18] V. Guinot and C. Delenne. MUSCL schemes for the shallow water sensitivity equations with passive scalar transport. Computers and Fluids, 59:11-30, 2012.

[19] J. Herman and W. Usher. Salib: an open-source python library for sensitivity analysis. The Journal of Open Source Software, 2(9), 2017.

[20] J. Hou, Q. Liang, F. Simons, and R. Hinkelmann. A 2D wellbalanced shallow flow model for unstructured grids with novel slope source term treatment. Advances in Water Resources, 52:107-131, feb 2013.

[21] J. Hou, F. Simons, M. Mahgoub, and R. Hinkelmann. A robust wellbalanced model on unstructured grids for shallow water flows with wetting and drying over complex topography. Computer Methods in Applied Mechanics and Engineering, 257:126-149, apr 2013.

[22] J. Hudson and P. K. Sweby. Formulations for numerically approximating hyperbolic systems governing sediment transport. Journal of Scientific Computing, 19(1-3):225-252, 2003.

[23] C. Juez, J. Murillo, and P. García-Navarro. A 2D weakly-coupled and efficient numerical model for transient shallow flow and movable bed. Advances in Water Resources, 71:93-109, 2014. 
[24] P. Y. Julien and B. Saghafian. Casc2d user's manual. Civil Engineering report, Dept. of Civil Engineering, Colorado State University, Fort Collins, CO, 80523, 1991.

[25] D. Kandel, A. Western, R. Grayson, and H. Turral. Process parameterization and temporal scaling in surface runoff and erosion modelling. Hydrological Processes, 18(8):1423-1446, 2004.

[26] Q. Liang. Flood Simulation Using a Well-Balanced Shallow Flow Model. Journal of Hydraulic Engineering, 136(9):669-675, sep 2010.

[27] Q. Liang. A coupled morphodynamic model for applications involving wetting and drying. Journal of Hydrodynamics, Ser. B, 23(3):273-281, 2011.

[28] Q. Liang and A. G. Borthwick. Adaptive quadtree simulation of shallow flows with wet-dry fronts over complex topography. Computers \& Fluids, 38(2):221-234, feb 2009.

[29] Q. Liang and F. Marche. Numerical resolution of well-balanced shallow water equations with complex source terms. Advances in Water Resources, 32(6):873-884, 2009.

[30] Q. Liang and F. Marche. Numerical resolution of well-balanced shallow water equations with complex source terms. Advances in Water Resources, 32(6):873-884, jun 2009.

[31] Q. H. Liang, J. Li, and S. Fu. A New Coupled Model for Simulating Shallow Flow Driven Morphological Change. 319:319-322, 2011. 
[32] X. Liu, B. Landry, and M. Garcia. Two-dimensional scour simulations based on coupled model of shallow water equations and sediment transport on unstructured meshes. Coastal Engineering, 55(10):800-810, 2008.

[33] R. Marsooli and W. Wu. Three-dimensional numerical modeling of dam-break flows with sediment transport over movable beds. Journal of Hydraulic Engineering, 141(1):04014066, 2014.

[34] E. Meyer-Peter and R. Müller. Formulas for bed-load transport. In IAHSR 2nd meeting, Stockholm, appendix 2. IAHR, 1948.

[35] J. Murillo and P. García-Navarro. An Exner-based coupled model for two-dimensional transient flow over erodible bed. Journal of Computational Physics, 229(23):8704-8732, nov 2010.

[36] J. Murillo and P. García-Navarro. Weak solutions for partial differential equations with source terms: Application to the shallow water equations. Journal of Computational Physics, 229(11):43274368, 2010.

[37] C. Paola and V. R. Voller. A generalized Exner equation for sediment mass balance. Journal of Geophysical Research: Earth Surface, 110(December 2004):1-8, 2005.

[38] A. Saltelli, D. Albrecht, S. Tarantola, and F. Ferretti. A new sample-based algorithms to compute the total sensitivity index. arXiv preprint arXiv:1703.05799, 2017.

[39] F. Simons, T. Busse, J. Hou, I. Özgen, and R. Hinkelmann. A model for overland flow and associated processes within the Hydroinfor- 
matics Modelling System. Journal of Hydroinformatics, pages 1$26,2014$.

[40] G. Simpson and S. Castelltort. Coupled model of surface water flow, sediment transport and morphological evolution. Computers \& Geosciences, 32(10):1600-1614, dec 2006.

[41] G. M. Smart. Sediment Transport Formula for Steep Channels. Journal of Hydraulic Engineering, 110(3):267-276, 1984.

[42] S. Soares-Frazão, R. Canelas, Z. Cao, L. Cea, H. M. Chaudhry, A. Die Moran, K. El Kadi, R. Ferreira, I. F. Cadórniga, N. GonzalezRamirez, M. Greco, W. Huang, J. Imran, J. Le Coz, R. Marsooli, A. Paquier, G. Pender, M. Pontillo, J. Puertas, B. Spinewine, C. Swartenbroekx, R. Tsubaki, C. Villaret, W. Wu, Z. Yue, and Y. Zech. Dam-break flows over mobile beds: experiments and benchmark tests for numerical models. Journal of Hydraulic Research, 50(4):364-375, aug 2012.

[43] S. Soares-Frazão and Y. Zech. Hllc scheme with novel wave-speed estimators appropriate for two-dimensional shallow-water flow on erodible bed. International Journal for Numerical Methods in Fluids, 66(8):1019-1036, 2011.

[44] S. Soares-Frazão. Experiments of dam-break wave over a triangular bottom sill. Journal of Hydraulic Research, 45(sup1):19-26, 2007.

[45] L. Song, J. Zhou, J. Guo, Q. Zou, and Y. Liu. A robust well-balanced finite volume model for shallow water flows with wetting and dry- 
ing over irregular terrain. Advances in Water Resources, 34(7):915932, 2011.

[46] R. Soulsby. Dynamics of marine sands: a manual for practical applications. Thomas Telford, 1997.

[47] T. Tingsanchali and C. Chinnarasri. Numerical modelling of dam failure due to flow overtopping. Hydrological Sciences Journal, 46(1):113-130, 2001.

[48] E. F. Toro. Shock-capturing Methods for Free-surface Shallow Flows. John Wiley \& Sons, New York/Chichester, 2001.

[49] E. F. Toro. Riemann Solvers and Numerical Methods for Fluid Dynamics. Springer-Verlag, Berlin Heidelberg, 3 edition, 2009.

[50] E. F. Toro, M. Spruce, and W. Speares. Restoration of the contact surface in the HLL-Riemann solver. Shock Waves, 4(1):25-34, jul 1994.

[51] B. van Leer. Towards the ultimate conservative difference scheme. V. A second-order sequel to Godunov's method. Journal of Computational Physics, 32(1):101-136, jul 1979.

[52] L. C. Van Rijn. Sediment transport, part i: bed load transport. Journal of Hydraulic Engineering, 110(10):1431-1456, 1984.

[53] D. Water and Environment. Mike11, a computer based modeling system for rivers and channels: Reference manual. 1995.

[54] K. C. Wilson. Analysis of bed-load motion at high shear stress. Journal of Hydraulic Engineering, 113(1):97-103, 1987. 
[55] W. Wu. Computational river dynamics. Taylor \& Francis, 2008.

[56] W. Wu and M. Asce. of Unsteady Flow and Nonuniform Sediment Transport in Open Channels. Database, 130(October):1013-1024, 2004.

[57] W. Wu and R. Marsooli. A depth-averaged 2D shallow water model for breaking and non-breaking long waves affected by rigid vegetation. Journal of Hydraulic Research, 50(6):558-575, dec 2012.

[58] W. Wu, R. Marsooli, and Z. He. Depth-averaged two-dimensional model of unsteady flow and sediment transport due to noncohesive embankment break/breaching. Journal of Hydraulic Engineering, 138(6):503-516, 2011.

[59] W. Wu and S. Wang. One-Dimensional Modeling of Dam-Break Flow over Movable Beds. Journal of Hydraulic Engineering, 133(January):48-58, 2007.

[60] W. Wu and S. S. Wang. One-dimensional explicit finite-volume model for sediment transport. Journal of Hydraulic Research, 46(1):87-98, 2008.

[61] J. Xia, B. Lin, R. A. Falconer, and G. Wang. Modelling dam-break flows over mobile beds using a $2 \mathrm{~d}$ coupled approach. Advances in Water Resources, 33(2):171-183, 2010.

[62] R. Zhang and J. Xie. Sedimentation research in China: Systematic selections. China and Power Press, 1993.

[63] J. Zhao, I. Özgen, D. Liang, and R. Hinkelmann. Comparison of depth-averaged concentration and bed load flux sediment trans- 
port models of dam-break flow. Water Science and Engineering, pages 287-294, 2017.

[64] J. Zhao, I. Özgen, D. Liang, and R. Hinkelmann. Improved multislope muscl reconstruction on unstructured grid for shallow water equations. International Journal for Numerical Methods in Fluids, 2018. fld.4499. 